\title{
DRUMLINLANDSCHAFT AUF DER WASSERSCHEIDE ZWISCHEN PIEKSÄMÄKI UND HAUKIVUORI IN MITTELFINNLAND
}

\author{
GunNar GlüCkert
}

\begin{abstract}
GlüCKeRt, GunNar 1971: Drumlinlandschaft auf der Wasserscheide zwischen Pieksämäki und Haukivuori in Mittelfinnland. Bull. Geol. Soc. Finland 43, $141-161$.
\end{abstract}

\begin{abstract}
A stereoscopic examination of 36 vertical air photographs at the scale $1: 60000$, in conjunction with the new topographic maps with contour lines at the 1:10 000 covering about $2450 \mathrm{~km}^{2}$ of Central Finland near Pieksämäki, has been carried out glacialmorphologically. The presence of a large field of several thousand glacial drumlins, of which about 1050 were linear parallel drumlins of the type »elongated ridges», was revealed and recorded. Some drumlin areas with narrow ridges between Haukivuori, Juva and Joroinen resemble the fluted moraine surface. The results of this study are shown in figure 1. In Central Finland the drumlins, $0.2-7 \mathrm{~km}$ in length, $0.05-1.5 \mathrm{~km}$ in width, $5-70 \mathrm{~m}$ in height, and with a width to length ratio of about $1: 4-$ $1: 8$, are very common and prominent in the landscape. They have been formed at high pressure both under the last inland glaciation and under the front of thinned ice during the movement of the last ice lobe which occupied the area. According to the investigations, both erosion (shaping) and accumulation of existing deposits occur.
\end{abstract}

Gunnar Glückert, Institut für Quartärgeologie, Universität Turku, 20500 Turku 50, Finnland.

\section{Einleitung}

In der quartärgeologischen Literatur erscheinen die ersten Angaben über Drumlins oder drumlinartige Moränen schon vor über 100 Jahren. Das aus dem Keltischen stammende Wort Drumlin in seiner ursprünglichen Bedeutung »kleiner Hügel» benutzte als erster H. M. Close (1866) für die Moränenbuckel Irlands (vgl. auch z.B. Fairchild 1907 S. 392). Ausser im skandinavischen Vereisungsgebiet gibt es Drumlinbildungen in grossen Feldern auch z.B. in den Vereinigten Staaten und Kanada, auf den briti- schen Inseln und Irland, in Deutschland, Polen, Russland und China (z.B. Ebers 1926, 1937, Charlesworth 1957 S. 391-393, Woldstedt 1961 S. 97, Embleton und King 1968 S. 333-335 und Thornbury 1969 S. 382).

Die finnische naturwissenschaftliche Literatur ist nicht sehr reich an Untersuchungen über Drumlinbildungen. Diese Moränenform ist wenig beachtet worden, da sie in Gegenden vorkommt, über die topographische Spezialkarten bis jetzt nicht publiziert worden sind. Kurze Erwähnungen und Untersuchungen über 
Drumlins liegen in etwa 30 Veröffentlichungen vor. Drumlinbildungen finden sich u.a. auf Åland (Jaatinen 1952, 1960 und Hausen 1964), in Häme und Mittelfinnland (Sederholm 1889, Frosterus 1913, Suomenmaa VI 1924, Hellaakoski 1934, Brander 1934, Korpela 1954, Virkkala 1961, 1969 und Aartolahti 1966, 1968), Ostfinnland (Rosberg 1892, Virkkala 1948, 1960 und Repo 1957), Ostbottnien (Leiviskä 1907, Tolvanen 1917, Okko 1949 sowie Mölder und Salmi 1954), Kainuu, Kuusamo und Südlappland (Hänninen 1915, Tanner 1938, Virkkala 1951, Kurimo 1964 und Kujansuu 1969).

Die landschaftliche Physiognomie der Gegend von Pieksämäki-Haukivuori ist vor allem durch Moränenrücken von Drumlinhabitus stark beeinflusst. Die ersten Beschreibungen der kargen Natur und monotonen Landschaft in der Gegend von Pieksämäki liefert Sederholm (1889 S. 4247). In seiner Reiseschilderung berichtet er auch über die glazialen Bildungen längs der Wasserscheide zu beiden Seiten der Eisenbahn zwischen Haukivuori und Pieksämäki.

Hult (1893 S. 38 - 40) beschreibt den Wasserscheidencharakter der Gegend von Pieksämäki und ihre Buckellandschaft und stellt fest, dass auf der nord-südlich gerichteten Wasserscheide viele abflusslose Seebecken liegen. Frosterus (1913 S. 6, 26) spricht in der Erläuterung zur Bodenkarte von Mikkeli von einer typischen Buckellandschaft im Gebiet von Mikkeli und den Kirchspielen Haukivuori, Pieksämäki und Juva. Im Band VI von Suomenmaa (1924 s. 113-149) erzählt der Text von streifiger Landschaft mit Moränenrücken in den Kirchspielen Pieksämäki, Haukivuori, Juva, Virtasalmi, Jäppilä und Joroinen. (Tanner (1938 S. 490) erwähnt von der schönen Drumlinlandschaft in der Gegend von Pieksämäki. Korpela (1954) legt in seiner unveröffentlichten geographischen Laudaturarbeit die Geomorphologie von Pieksämäki und dessen Nahumgebung dar und beschreibt verhältnismässig eingehend den Felsgrund sowie den Boden des Gebiets und seine langen Moränenrücken.

\section{Untersuchungsgebiet}

Ungefähr N-S-gerichtet verläuft durch das Seegebiet Mittelfinnlands der sog. Savonselkä in einer aus Hügeln und Mooren bestehenden Landengenzone von NW-seits von Kuopio bis S-seits von Mikkeli. Diese höher als die sie umgebenden Gebiete gelegene seenarme Wasserscheide trennt das Gewässergebiet des PäijänneSees von den in den Saimaa-See abfliessenden Gewässern. Die Wasserscheide zwischen den Gewässern des Saimaa und denen des Päijänne ist schwer genau zu umgrenzen, aber ihr Verlauf folgt ungefähr der Eisenbahn Mikkeli-Pieksämäki (vgl. Sederholm 1889 S. 42-44, Frosterus 1913 S. 9 und Suomenmaa VI 1924 S. 114, 117). In der Erstreckung der Wasserscheide lassen sich zwei Hauptrichtungen unterscheiden, von denen NNW-SSO dem Streichen der parallelen Felsund Moränenrücken nachgeht und SW-NO durch ausgedehnte, ebene Moorflächen gekennzeichnet ist. Die Breite der Wasserscheide wechselt zwischen etwa 200 Metern und vielen Kilometern (vg1. Korpela 1954) (Abb. 1).

Das Untersuchungsgebiet liegt in Mittelfinnland zwischen Pieksämäki und Haukivuori zu ihren beiden Seiten in der Mitte der Wasserscheide. Zum eigentlichen Untersuchungsgebiet, das beinahe ganz im Län Mikkeli gelegen ist, gehören ausser Stadt- und Landgemeinde Pieksämäki Teile der Landgemeinde Mikkeli sowie der Nachbarkirchspiele Jäppilä, Joroinen, Juva, Virtasalmi, Haukivuori, Kangasniemi und Hankasalmi. Es ist versucht worden, das erforschte Gebiet, dessen Grösse rd. $40 \times 60 \mathrm{~km}$ und dessen Flächeninhalt $2450 \mathrm{~km}^{2}$ ausmacht, so zu wählen, dass der Hauptteil des weiten Drumlinfeldes von Mittelfinnland einbezogen wird (Abb. 1).

Zweck dieser Untersuchung ist es gewesen, anhand von Luftaufnahmen, die neuerschienenen topographischen Teilkarten (1:10 000) der noch unpublizierten Grundkarten und Forschungen im Felde im Sommer 1970 die landschaftbildenden »Selkä-Rücken» aufzuzeigen sowie glazialmorphologisch und genetisch zu erhellen. Die 


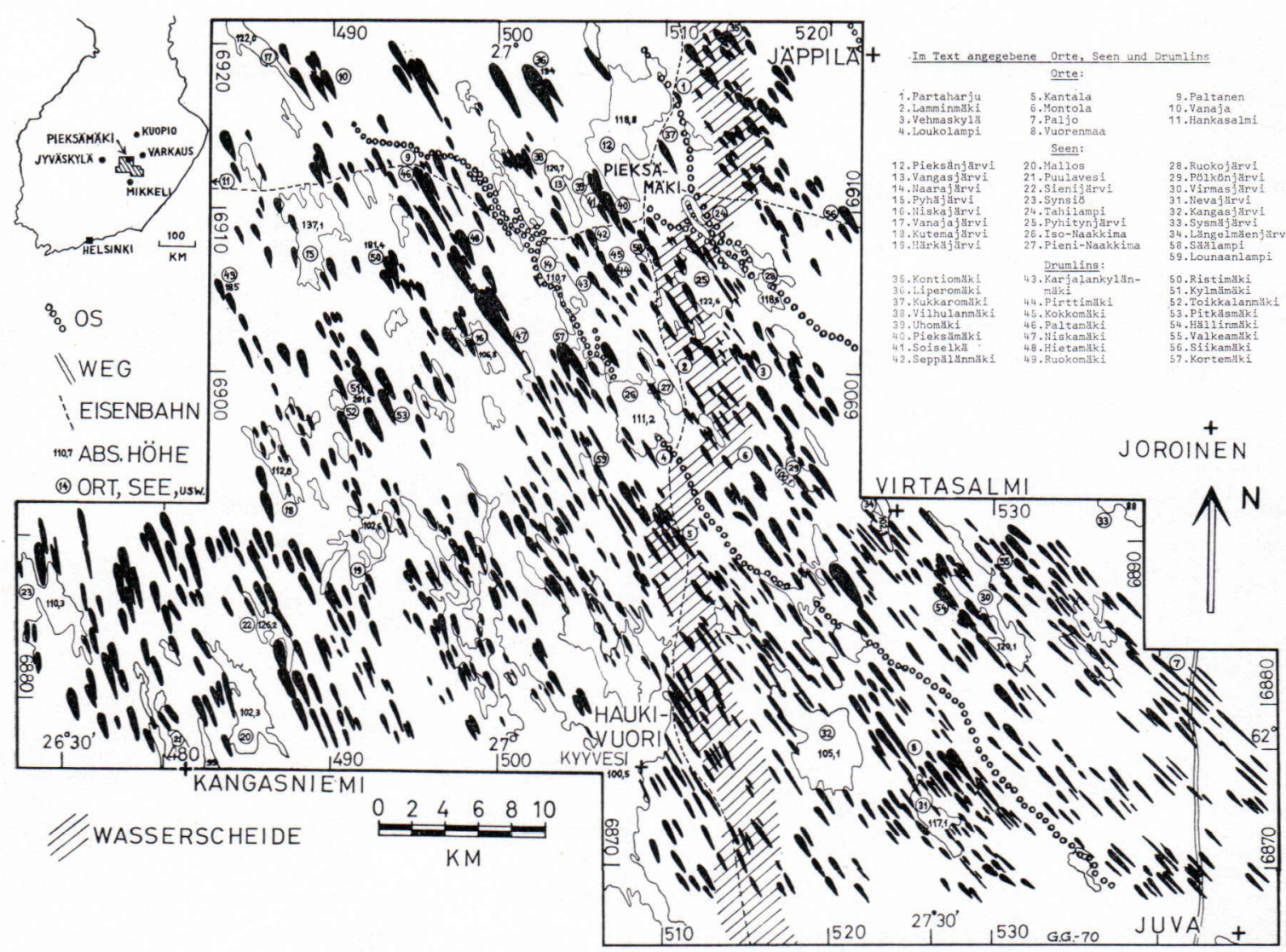

Abb. 1. Karte vom Untersuchungsgebiet in der Gegend, die von Pieksämäki, Hankasalmi, Kangasniemi, Haukivuori, Juva, Joroinen und Jäppilä begrenzt ist und in der 1050 Drumlins mit fächerförmiger Ausstrahlung in dem grossen Drumlinfeld Mittelfinnlands vorkommen. Diese Moränenrücken verleihen durch ihr geselliges Auftreten der Landschaft einen ausgeprägt streifigen Charakter.

Rückenbildungen sind auf den Karten und Luftaufnahmen ausserordentlich gut von ihrer flachen Umgebung zu unterscheiden. Das umfangreiche Drumlinfeld in Mittelfinnland umfasst im ganzen rd. 2000-3000 Rücken, von denen etwa $1050 \mathrm{im}$ Untersuchungsgebiet anzutreffen sind. Die Aufgabe einer künftigen Untersuchung ist, ausführlicher die Formtypen und Entstehung der Moränenrücken sowie, sobald neue topographische Karten auch weiter östlich publiziert werden, die Ausbreitung des ganzen Drumlinfeldes in Mittelfinnland aufzuklären.

Die Umgebung der Stadt Pieksämäki ist sowohl mit Hilfe von 16 topographischen Kar- ten, nämlich Nr. 323202 A-D (Naarajärvi), 323203 A-D (Pieksämäki), 323205 A-D (Pyhitty), 321411 C-D (Niskakoski) und $321412 \mathrm{C}$ (Paltanen), als auch mittels 36 Luftaufnahmen (1:60 000) erforscht worden. Bei dem übrigen Untersuchungsgebiet sind, ausser einer Karte von Virtasalmi, Nr. 323109 D (Hällinmäki), die länglichen Moränenrücken stereoskopisch nur aus den Luftaufnahmen ermittelt worden.

In der Seenplatte Mittelfinnlands liegt die Gegend von Pieksämäki-Haukivuori auf einem verhältnismässig ebenen, moorreichen Wasserscheidegebiet. Sie liegt mit einer absoluten Durchschnittshöhe von $120 \mathrm{~m}$ deutlich höher 
TABElle 1.

Die absoluten und relativen Höhenverhältnisse in der Gegend von Pieksämäki

\begin{tabular}{|c|c|c|c|c|c|}
\hline & Kartenblatt & $\begin{array}{l}\text { Abs. Höhe } \\
\text { Max. Min. }\end{array}$ & $\begin{array}{c}\text { Relat. Höhe } \\
\text { Max. Durchschn. }\end{array}$ & $\begin{array}{l}\text { Abs. Höhe der } \\
\text { Niederungen } \\
\text { (Seen, Süpfe) }\end{array}$ & Grundkarte \\
\hline $\begin{array}{r}323202 \mathrm{~A} \\
\mathrm{~B} \\
\mathrm{C} \\
\mathrm{D}\end{array}$ & 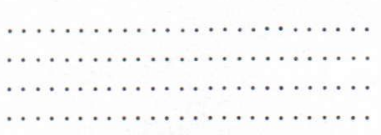 & $\begin{array}{ll}154 & 107 \\
149 & 111 \\
156 & 111 \\
196 & 111\end{array}$ & $\begin{array}{ll}47 & 10-25 \\
38 & 10-20 \\
45 & 10-20 \\
85 & 15-40\end{array}$ & $\begin{array}{l}107-120 \\
111-125 \\
111-125 \\
111-140\end{array}$ & Naarajärvi \\
\hline $\begin{array}{r}323203 \text { A } \\
\text { B } \\
\text { C } \\
\text { D }\end{array}$ & 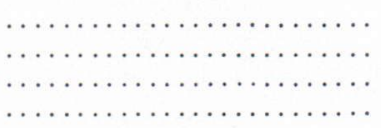 & $\begin{array}{l}113 \\
117 \\
119 \\
119\end{array}$ & $\begin{array}{l}10-20 \\
10-30 \\
10-30 \\
10-20\end{array}$ & $\begin{array}{l}113-125 \\
117-140 \\
119-125 \\
119-130\end{array}$ & Pieksämäki \\
\hline $\begin{array}{r}323205 \text { A } \\
\text { B } \\
\text { C } \\
\text { D }\end{array}$ & 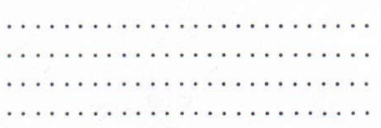 & $\begin{array}{ll}160 & 111 \\
152 & 118 \\
167 & 110 \\
166 & 112\end{array}$ & $\begin{array}{l}10-20 \\
10-20 \\
10-30 \\
10-20\end{array}$ & $\begin{array}{l}111-130 \\
118-130 \\
110-130 \\
112-130\end{array}$ & Pyhitty \\
\hline $\begin{array}{rl}321411 & \mathrm{C} \\
\mathrm{D}\end{array}$ & $\begin{array}{c}\cdots \cdots \cdots \cdots \cdots \cdots \cdots \cdots \cdots \cdots \\
\cdots \cdots \cdots \cdots \cdots \cdots \cdots\end{array}$ & $\begin{array}{l}106 \\
108\end{array}$ & $\begin{array}{l}10-20 \\
10-20\end{array}$ & $\begin{array}{l}106-125 \\
108-125\end{array}$ & Niskakoski \\
\hline $321412 \mathrm{C}$ & $\ldots \ldots \ldots \ldots \ldots \ldots \ldots \ldots \ldots$ & 113 & $10-30$ & $113-135$ & \multirow[t]{2}{*}{ Paltanen } \\
\hline \multicolumn{2}{|c|}{ Durchschnitt: $\ldots \ldots \ldots \ldots \ldots \ldots \ldots$} & 112 & $10-24$ & $112-129$ & \\
\hline $323109 \mathrm{D}$ & $\ldots \ldots \ldots \ldots \ldots \ldots \ldots \ldots \ldots$ & 164 & $10-25$ & $98-125$ & Hällinmäki \\
\hline
\end{tabular}

als die topographisch abwechslungsreichere Umgebung rings um die Städte Mikkeli, Jyväskylä, Kuopio, Varkaus und Savonlinna.

Die absoluten Höhenverhältnisse in der Gegend von Pieksämäki variieren nach den 16 benutzten Karten zwischen 105 und 195 m (durchschn. min. $112 \mathrm{~m}$, max. $164 \mathrm{~m}$ ) und die relativen zwischen 10 und $85 \mathrm{~m}$ (durchschn. min 15, max. $52 \mathrm{~m}$ ), ausser den Rücken der Drumlins aber oft nur zwischen 10 und $25 \mathrm{~m}$. Die Seen und Moorniederungen liegen in der Gegend von Pieksämäki durchschnittlich in 112-129 m ü.d.M. Nach Renkonen (1933 S. 16) beträgt die Mittelhöhe in Seen-Finnland im Kleinhügelland von Gross-Savo zwischen 114,6 und 116,6 m ü.d.M. Auf der Wasserscheide sind die relativen Höhenunterschiede stellenweise also sehr unbedeutend. Die Drumlins bilden die einzigen hohen Rücken in der ebenen Landschaft und verleihen der Topographie ein fremdes Gepräge (Tabelle 1).

Die höchste Stelle im Untersuchungsgebiet liegt auf dem Tornikangas in der Nähe von
Vanaja bei 218,7 $\mathrm{m}$ und die niedrigste ist der See Sysmäjärvi unweit Joroinen bei $88 \mathrm{~m}$ ü.d.M. Besonders hohe und breite Drumlinrücken sind u.a. Liperomäki (194 m ü.d.M.), Paltamäki (178 m), Hietamäki (166 m), Niskamäki (164 m), Ruokomäki (185 m), Hällinmäki (164 m), Kokkomäki (196 m), Seppälänmäki (174 m), Uhomäki (160 m), Ristimäki (181 m), Pitkäsmäki (175 m), Toikkalanmäki (172 m) und Kylmämäki (201,5 m). (Abb. 1.).

Das Wasserscheidegebiet ist in Mittelfinnland die seenärmste Gegend, deren Seenprozent rd. $10-30 \%$, NW-seits von Pieksämäki stellenweise bis unter $5 \%$ vom Gesamtflächenraum des Gebiets ausmacht. Das Drumlingebiet Mittelfinnlands umrandet viele grosse Seen, wie Puulavesi (95 m ü.d.M.), Synsiä (110 m), Sysmäjärvi (88 m), Syvävesi (102 m), Längelmäenjärvi (99 m) und Kyyvesi (101 m). Die Wasserspiegel der Seebecken im Untersuchungsgebiet selbst, bei $110-130 \mathrm{~m}$, liegen durchschnittlich $20-40 \mathrm{~m}$ höher als die der umliegenden grossen Seen 
Mittelfinnlands. Daher fliesst das Wasser von der Wasserscheide in allen Himmelsrichtungen ab. Grosse Seen in der Umgebung des Untersuchungsgebiets sind Unnukka (81 m), Haukivesi (76 m), Kyyvesi (101 m), Puulavesi (95 m), Hankavesi (96 m), Armisvesi (106 m), Konnevesi $(95 \mathrm{~m})$, Suonteenselkä $(99 \mathrm{~m})$ und Sorsavesi $(98 \mathrm{~m})$.

\section{Felsgrund, Erboden und Oberflächengestaltung}

Der Felsgrund des betreffenden Gebiets ist nicht sehr abwechslungsreich. Der Felsgrund im Gebiet von Pieksämäki wird als westlicher Teil zu der Gneis- und Migmatitzone gezählt, die in breiter, SO-NW-gerichteter Folge die karelischen Formationen von den Graniten Mittelfinnlands trennt. Nach Eskola (1941) haben die gefalteten migmatitischen Bildungen von Savo ihre gegenwärtige Hauptstreichrichtung im $\mathrm{Zu}$ sammenhang mit der karelischen Bergkettenfaltung erhalten.

Die häufigsten Gesteinsarten des Felsgrundes in der Gegend von Pieksämäki sind Granit und Granodiorit mit ihren Metamorphoseergebnissen sowie die jüngeren kristallinen Schiefer und Migmatite. Das Gebiet von Kangasniemi im Südwesten gehört ausschliesslich zu den Graniten Mittelfinnlands, in der Gegend von Haukivuori sind die Glimmer- und Adergneise sowie die älteren Granite kennzeichnend, in Virtasalmi umgeben die alte weite Granitintrusion glimmerschieferartige Gneise und Hornblendeschiefer, die von Juva bis Jäppilä reichen (vgl. Frosterus 1902 und Wilkman 1938).

Felsblössen gibt es im Untersuchungsgebiet sehr spärlich. Das Grundgebirge tritt in Felshöcker nur an etwa 2-4 Stellen von je $10 \mathrm{~km}^{2}$ aus der Moränen- und Torfdecke hervor. Der Felsgrund mit weichen schieferartigen Gesteinsarten ist in weiten Flächen von Moränen und anderen quartären Ablagerungen überlagert, weshalb nackte Felsen mit Schrammen sehr selten sind. In neuen Schnitten der Weganlagen und Eisenbahn kommen ausmodellierte Rund-

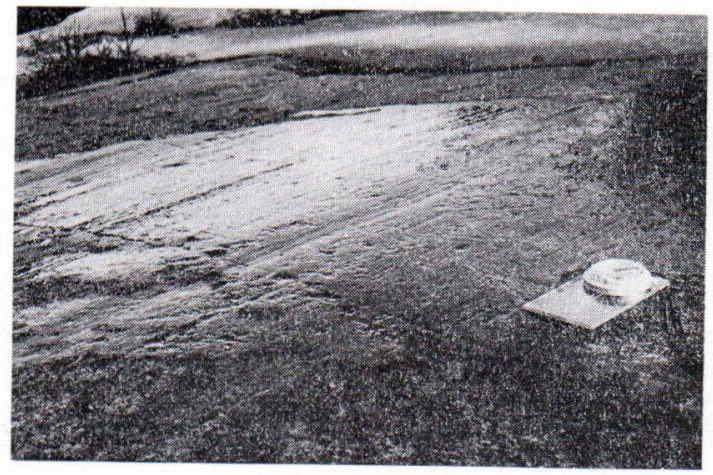

Abb. 2. Zwischen Naarajärvi und Säälampi in der Stadt Pieksämäki ist beim Strassenbau ein kleiner geschliffener Felshöcker aus Glimmeradergneis zum Vorschein gekommen. Die geschrammte Oberfläche $\left(325^{\circ}\right)$ von W aus gesehen. Kordinaten der Beobachtungsstelle: 69 0836/5 0828. Photo Glückert 1. 8. 1970.

höckerkomplexe mit feingeschliffener und gestreifter Oberfläche zum Vorschein (vgl. Sederholm 1889 S. 45). (Abb. 2).

Das Inlandeis hat sich in diesen Gegenden, nach den wenigen Schrammenbeobachtungen zu schliessen, in der Richtung NW-SO vorwärtsbewegt. In den verschiedenen Teilen des Untersuchungsgebiets treten in den Richtungen der Schrammen verhältnismässig deutliche Unterschiede $\left(345-310^{\circ}\right)$ hervor. In den Westteilen wechseln die Richtungen von $345-330^{\circ}$, von wo aus die Zahlenwerte gleichmässig gegen Osten bis auf $320-310^{\circ}$ abnehmen. Deutliche Kreuzschrammen sind im Gebiet von Pieksämäki nicht aufgefunden worden (vgl. Sederholm 1889 S. 4 und Frosterus 1913 S. 19-21). (Abb. 2).

Das Gelände in Mittelfinnland ist zum grössten Teil eine Decke von mächtiger, hartgepackter Grundmoräne und dünner Moorschicht. Von Moräne sind auch Moor- und Sandböden unterlagert. Moräne ist ziemlich gleichmässig auf das ganze Gebiet verteilt und kommt entweder als schmale, von Mooren umgenene Rücken oder in einigen höher gelegenen Gebieten als einheitliche Moränenfläcken vor.

Die Mächtigheit der Moränenmassen wechselt im allgemeinen zwischen 1 und $20 \mathrm{~m}$ und dürfte im Mittel von der Grössenordnung 2-5 m sein. 
Die grossen Moränenrücken umfassen oft mächtige Massen, aber an den proximalen Enden der Drumlins tritt der feste Gesteinskern oft schon in 1-3 m Tiefe zutage. Da der Moränenmantel im allgemeinen jedoch verhältnismässig dünn ist, bildet seine Oberfläche in ihren Hauptzügen die Topographie des Felsgrundes nach. In Mittelfinnland sind die Höhenschwankungen, mit Ausnahme der Drumlins, so gering, dass die Moräne die meisten Unebenheiten des Reliefs auszugleichen vermocht hat.

Zwei lange, unterbrochene, 5-25 $\mathrm{m}$ hohe Oszüge mit wohlentwickeltem Rücken-, Heideund Sollgelände ziehen schräg durch das Untersuchungsgebiet von Juva durch Kantala, IsoNaakkima, Naarajärvi nach Paltanen und von Virtasalmi durch Tahilampi nach Pieksämäki und Partaharju (vgl. Sederholm 1889 S. 22-23, 25 Frosterus 1913 S. $34-35$ und Suomenmaa VI 1924 S. 118) und setzen sich weiter bis nach Pihtipudas fort (Brander 1934 S. 28) (Abb. 1).

Tonböden sind in dieser Gegend nicht anzutreffen. Die ebenen Niederungen und die bebauten Äcker auf den Drumlins bestehen aus SchluffFeinsand, die aus Grundmoräne herrühren.

Die Moore nehmen im Untersuchungsgebiet im Mittel $20 \%$ vom Flächeninhalt ein, am meisten NW von Pieksämäki (50\%), am wenigsten in den höchstgelegenen unebenen Gebieten (5$15 \%$ ). Die meisten Moore erscheinen in den Tälern als weite, netzartig verzweigte Moorkomplexe, Bruch-, Reiser- und Weissmoore. In vielen Gegenden wechseln miteinander parallel verlaufende Moränenrücken mit moorigen oder versumpften trogartigen Senken von ähnlicher Ausdehnung ab. Die langen Rücken sind Felsund Moränenhügel, die Talniederungen im allgemeinen Moore und Seen (Frosterus 1913 S. 6 und Korpela 1954).

Die gegenwärtige Topographie des Felsgrundes Finnlands ist entstanden durch endogene Einwirkungen, wie tektonische Dislokationen, Brüche und Faltungen, sowie durch exogene Faktoren, Verwitterung, Glazial- und Fluvialerosion. Von den runden Felskuppen aus Tiefen- gesteinen unterscheiden sich die flach geschwungenen Felsrücken aus Gneisen und anderen orientierten Gesteinen. Ihre Richtung befolgt im allgemeinen dem Verlauf der Schieferung.

Die Selektivität präglazialer Verwitterung erweist ein ausserordentlich intensives Abtragen des Verwitterungsvorgangs in die Intrusionskontakte. Die Kontaktzonen bilden im gegenwärtigen Relief Eintiefungen, in denen die Seen des Untersuchungsgebiets zu einem bedeutenden Teil Raum gefunden haben. Dabei befolgen sie zuweilen sogar konsequent die Richtung der von der Bewegungsroute des Inlandeises abweichenden Kontakte; diese Richtung zeichnet sich z.B. im Gebiet von Pieksämäki in den Kontakten zwischen Granodioriten und schieferigen Gneisen ab (vgl. Korpela 1954).

Die Unabhängigkeit der tektonischen Hauptbruchlinien des Felsgrundes von den Gesteinsund Schieferungslinien bezeugt, dass die ersteren wirkliche Verwerfungslinien sind. Dagegen hängen die schwächer entwickelten Querrichtungen mit der tektonischen Struktur des Felsgrundes zusammen und verlaufen in der Orientierung der Schieferung, insbesondere in den Kontaktzonen (Korpela 1954).

Die Entstehung der Spalt- und Bruchlinien vollzieht sich in den ursprünglich im Zusammenhang mit der Faltung erschienenen Schwächegürteln des Felsgrundes oder in Spannungszonen eruptiver Gesteine. Bei den orogenen Bewegungen gleichen sich die Spannungszustände der Erdrinde durch Faltung aus und entladen sich schliesslich durch Spaltung. Die jüngsten Bruchlinien dürften noch im Zusammenhang mit der Landhebung des Spätquartärs entstanden oder wenigstens aufs neue rissig geworden sein.

Die NW-SO verlaufende orographische Richtung ist beinahe überall in Mittelfinnland augenfällig. In der Gegend Pieksämäki-Haukivuori hat sich in der Entstehung der Streifung der Erdrinde der schieferige Felsgrund mit seinen typischen Spaltrichtungen ausgewirkt. Offenbar hat erst die Eiszeit diese vielleicht schon zu weitgehender Unkenntlichkeit herabgeminderte 
Streifungstendenz zutage zu bringen vermocht, denn die Streichrichtung der Gesteine fällt an vielen Stellen mit der Bewegungsrichtung des Inlandeises zusammen. Im allgemeinen haben sich die Züge des präglazialen Felsgrundes unter der Bodendecke ausgeglichen (Frosterus 1913 S. 5-6 und Korpela 1954).

Die Grosstopographie des Untersuchungsgebiets richtet sich nach dem präglazialen Relief des Felsgrundes. Die den uralten Felsgrund überlagernde junge Bodendecke bereichert das Gepräge der Kleintopographie in erster Linie um die Aufschüttungsformen von Moräne und glazifluvialen Massen. Ausser diesen gleichen auch die Torfablagerungen die Unebenheiten des Felsgrundes aus. Die formende Tätigkeit des ehemaligen Inlandeises kommt in der Landschaft überall zum Ausdruck. Eiszeitliche Glazialerosion und -akkumulation sowie die nacheiszeitlichen Meeres- und Seephasen wie auch Verwitterung haben überall im Untersuchungsgebiet die Kleintopographie vollendet.

Die deutliche Streifung der Erdoberfläche zeichnet sich landschaftlich am besten in der Ausrichtung der langen Fels- und Moränenrücken, der Moorfiguren und Seebecken ab (vgl. Sederhelm 1889 S. 46, Hult 1893 S. 38-40, Frosterus 1913 S. 6, 26, Hänninen 1915 S. 20-26 und Suomenmaa VI 1924 S. 113). Die Orientierung von Strandlinie, Buchten und Halbinseln der Seebecken ist präglazialen Ursprungs, denn die Streifungstendenz im Gelände hat in den Verwerfungs- und Schieferungsrichtungen des schon als Unterlage vorhandenen Felsgrundes verborgen gelegen, in Richtungen, die mit der Hauptbewegungsrichtung des Eises zusammenfallen. Die Richtung der Fels- und Moränenrücken ist durch die Eisbewegung mehr als durch die Gesteinsart und ihre Schieferung oder Schichtstellung bestimmt (Högbom 1905 S. 180 und Korpela 1954). Der Verfasser ist der Ansicht, dass die Drumlins oft besser als die Schrammen die zufälligen oder lokalen Veränderungen der Eisbewegung indizieren (vgl. Gillberg 1955 S. 513 und Flint 1957 S. 72).

\section{Dimensionen, Form und Ausbreitung der Drumlins}

Als Drumlins bezeichnet man flachgewölbte Grundmoränenformen, deren Längsachse in gleicher Richtung wie Eisbewegung und Gletscherschrammen verläuft. Zu den selbständigsten und am leichtesten wahrnehmbaren Aufschüttungsformen der Moränenmassen im Untersuchungsgebiet gehören die zahlreichen Drumlinrücken, die beträchtlich länger als breit sind.

Eine landschaftlich deutlich ausgeprägte Drumlintopographie mit gut ausgebildeten Rükken findet sich u.a. in Kontiomäki im Kirchspiel Jäppilä, in Paltanen, Vanaja, Kylmämäki und im Gebiet Pyhitynjärvi-Vehmaskylä in der Landgemeinde Pieksämäki, im Gebiet LoukolampiKantala und in Haukivuori im Kirchspiel Haukivuori, im Gebiet Virtasalmi-Hällinmäki und Valkeamäki im Kirchspiel Virtasalmi, am N-Ufer des Sees Kangasjärvi an der Grenze zwischen den Kirchspielen Haukivuori und Virtasalmi, im Gebiet Vuorenmaa und Kaikumäki im Kirchspiel Juva und im Gebiet Paljo im Kirchspiel Joroinen. (Abb. 1 und 3).

Die schönsten und am besten entwickelten Drumlinterrains der Inlandeisgebiete der letzten Eiszeit sind wahrscheinlich in den Vereinigten Staaten und Kanada zu finden. Z.B. im Lehrbuch der Geologie von A.J. Eardley gibt es ein Stereo-Luftaufnahmenpaar über eine ausserordentlich schöne Drumlinlandschaft in $\mathrm{Ka}$ nada (Eardley 1965 S. 230-231, Fig. 12-33).

Die Dimensionen der Drumlins im Untersuchungsgebiet schwanken innerhalb weiter Grenzen, mit Höhen von 5 bis $70 \mathrm{~m}$, Längen von $200 \mathrm{~m}$ bis $7 \mathrm{~km}$ und Breiten von $50 \mathrm{~m}$ bis $1500 \mathrm{~m}$. In Mittelfinnland sind wahrscheinlich die grössten Drumlins in Finnland und ganz Skandinavien anzutreffen. Zwar hat der Verfasser in der Gegend von Laukaa, Äänekoski, Saarijärvi und Sumiainen, etwa $100 \mathrm{~km} \mathrm{NW}$ vom Untersuchungsgebiet entfernt, noch höhere drumlinartige Rücken vom »Vaara-Typ», beobachtet (vgl. Högbom 1905 und Hänninen 1915 S. 6), die auch in der Richtung der letzten Eisbe- 


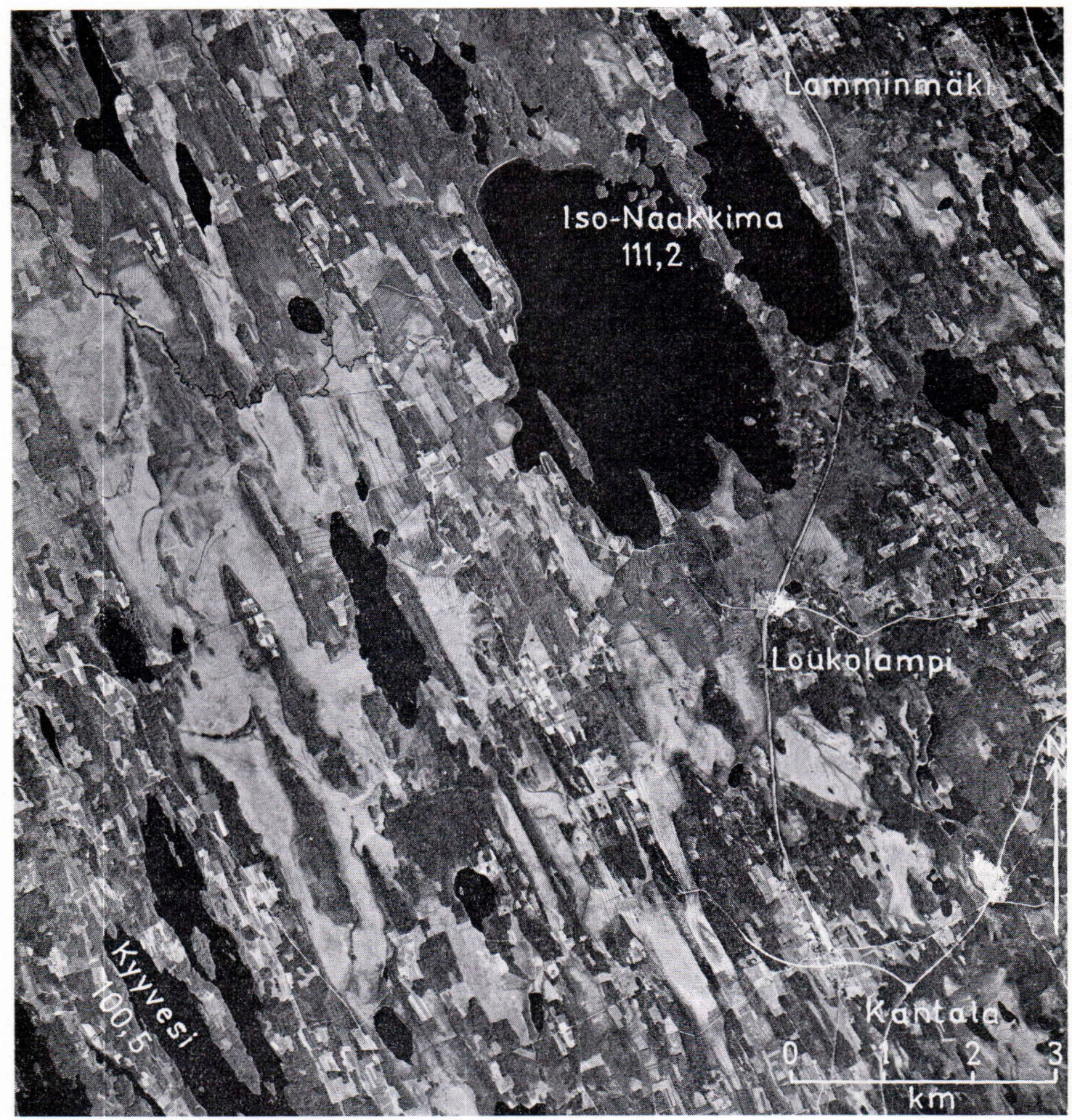

Abb. 3. Streifige Drumlinlandschaft mit schmalen, in die Länge gezogenen Rücken, meistens vom Typ 4, in der Gegend von Lamminmäki, Loukolampi, und Kantala auf der Wasserscheide zwischen Pieksämäki und Haukivuori. Die Luftaufnahme wird mit Genehmigung des Topographischen Instituts der Finnischen Wehrmacht veröffentlicht.

wegung liegen. Die grössten Drumlins im Untersuchungsgebiets sind in den Gegenden von Liperomäki, Paltanen, Vanaja, Niskamäki und Kylmämäki und auch stellenweise anderswo (Virtasalmi) anzutreffen. Diese Rücken vom »SelkäTyp» mit ihrem hohen Proximalende sind 3 bis
$7 \mathrm{~km}$ lang und 30 bis $70 \mathrm{~m}$ hoch. (Abb. 4, 5 6, 9 und 13).

Anderorts in Finnland erstrecken sich etwa $1-50 \mathrm{~m}$ hohe und $0,1-4 \mathrm{~km}$ lange Drumlins, im Mittel $1-20 \mathrm{~m}$ und $0,1-2 \mathrm{~km}$, von denen die grössten im Suomenselkä, in Kainuu und 


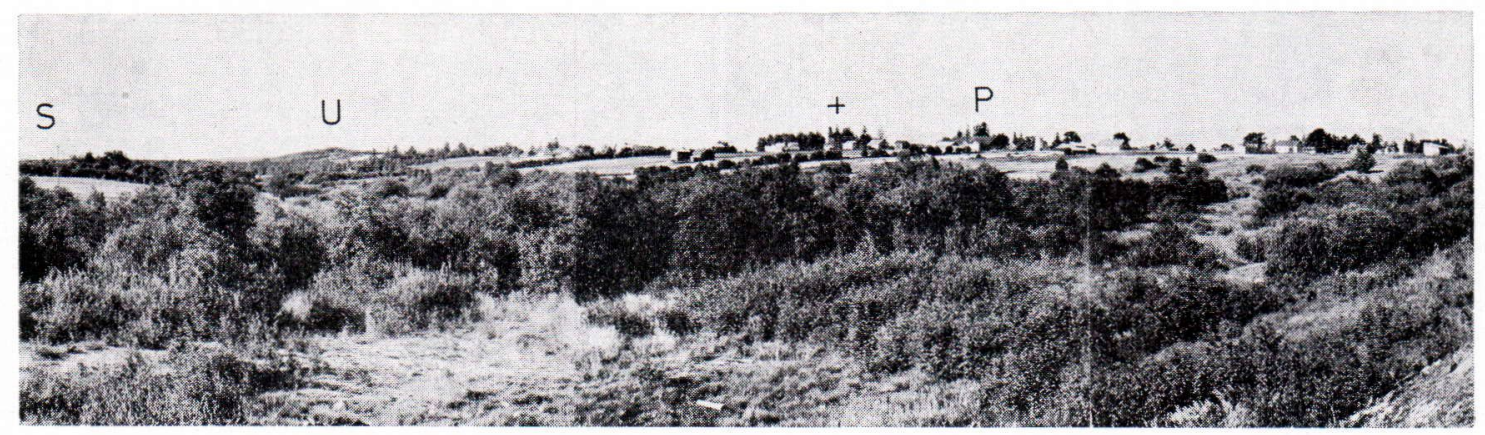

Abb. 4. Der Doppelrücken Uhomäki (U)-Pieksämäki (P) von dem kleinen Nachbardrumlin Soiselkä (S) aus gesehen. Die Kirche von Pieksämäki (+) in der Mitte. Vgl. Abb. 5 und auch Gillberg (1955 S. 514-515, Fig. 17). Photo Glückert 1. 8. 1970.

Kuusamo vorkommen dürften (Leiviskä 1907 S. 55-56, Hänninen 1915 S. 6, Tolvanen 1917 S. $103-104$, Sauramo 1928 S. 32-33, Brander 1934, Hellaakoski 1934, Tanner 1938 S. 487, Virkkala 1948 S. 26, 1951, 1961, S. 226-227, 1969 S. 11, 60, Jaatinen 1952 S. 57, 1960 S. 11 23, Mölder und Salmi 1954 S. 20-21, Okko 1949 S. 22-23, 1964 S. 260-262, Hausen 1964 S. 138, Aartolahti 1966 S. 43 und 1968 S. $23-$ 24).

In Schweden sind bis $\mathrm{zu} 40-70 \mathrm{~m}$ hohe und $5 \mathrm{~km}$ lange Drumlins angetroffen worden, aber durchschnittlich sind es $2-20 \mathrm{~m}$ hohe und 0,1-2 km lange Rücken (vgl. z.B. de Geer 1895 S. 212-213, Högbom 1905 S. 176, Sahlström 1909 S. 5-8, G. Lundqvist 1943 S. 21, Hoppe 1951 S. 158-160, 1959 S. 204, Björnsson 1953 S. 117-119, Gillberg 1955 S. 514, Björk 1959 S. $241-243$, Bergdahl 1961 S. 233-236, Persson 1966 S. 100 und J. Lundqvist 1970 S. 318-319). Anderswo, in Amerika, Deutschland und auf den britischen Inseln sind die Drumlins selten über $70 \mathrm{~m}$ hoch und über $5 \mathrm{~km}$ lang (z.B. Chamberlin 1883 S. 306,1894 S. 521 -523, Alden 1905 S. 19 , Fairchild 1907, 1929 S. 17-18, Ebers 1926, 1937, Hollingworth 1931, v. Klebelsberg 1948 S. 279282, Charlesworth 1957 S. 389, Flint 1957 S. 66, Wright 1957 S. 22, Lemke 1958 S. 278-281, Woldstedt 1961 S. 97-100, Holmes 1965 S. 663, Vernon 1966 S. 404 405, Embleton und King 1968 S. 322 und Thornbury 1969 S. 382).
Das Verhältnis zwischen Breite und Länge der Drumlins ist ebenfalls sehr variabel, denn es wechselt zwischen 1:2,5 und 1:15, und als Mittelwert dürfte 1:4-1:6 im nördlichen Teil des Untersuchungsgebiets und 1:5-1:8 in seinem südlichen Teil angeführt werden. Das Verhältnis ist also im Nordteil (Pieksämäki) geringer (1:4-1:12) als bei der Gegend von Loukolampi, Haukivuori, Virtasalmi und Juva (1:5-1:15) (Abb. 7).

In dem genannten Südteil des Untersuchungsgebiets sind die Drumlins stellenweise sehr lang, schmal und niedrig, ohne ein rundliches, hohes Proximalende. Das Terrain mit besonders langen Streifen (1:10-1:15) ähnelt dem »fluted moraine surface- oder fluting-Typus» (vgl. z.B. Fairchild 1929 S. $16-17$, Hoppe und Schytt 1953, Flint 1957 S. $69-71$, Lemke 1958 S. $281-282$, Gravenor und Meneley 1958 S. 715-719, Embleton und King 1968 S. 326 sowie Kujansuu 1969 S. 1-5) und könnte auf eine starke Neigung des Eises zur Drumlinbildung hindeuten (vgl. Abb. 1).

Die bekannteste Typeneinteilung der Drumlins geht auf Chamberlin zurück, der als erster von »linear oder elongated ridges, elliptical hills und mamillary hills» schreibt (Chamberlin 1883 S. 306 und 1894 S. 522). Rosberg (1892 S. 39, 120) teilt die Drumlins Ostfinnlands in drei Typen ein, von denen der häufigste der Rückentyp mit länglichem Felskern und länglicher Form 


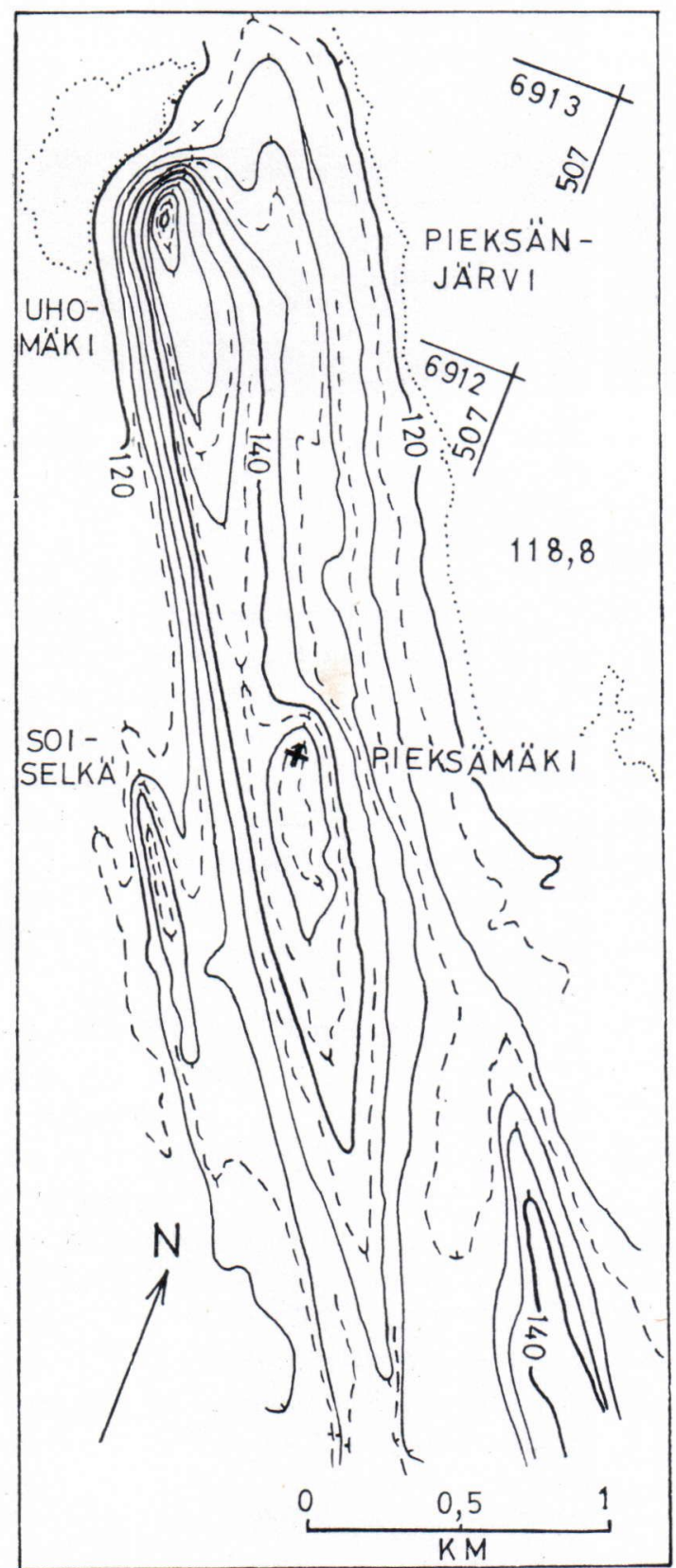

Abb. 5. Der $4 \mathrm{~km}$ lange Doppelrücken UhomäkiPieksämäki besteht aus zwei hintereinandergelegenen Drumlins (vgl. Abb. 4). Verkleinerte Kopie aus den topographischen Karten, 1 : 10 000, Nr. 323202 D und $323203 \mathrm{C}$.

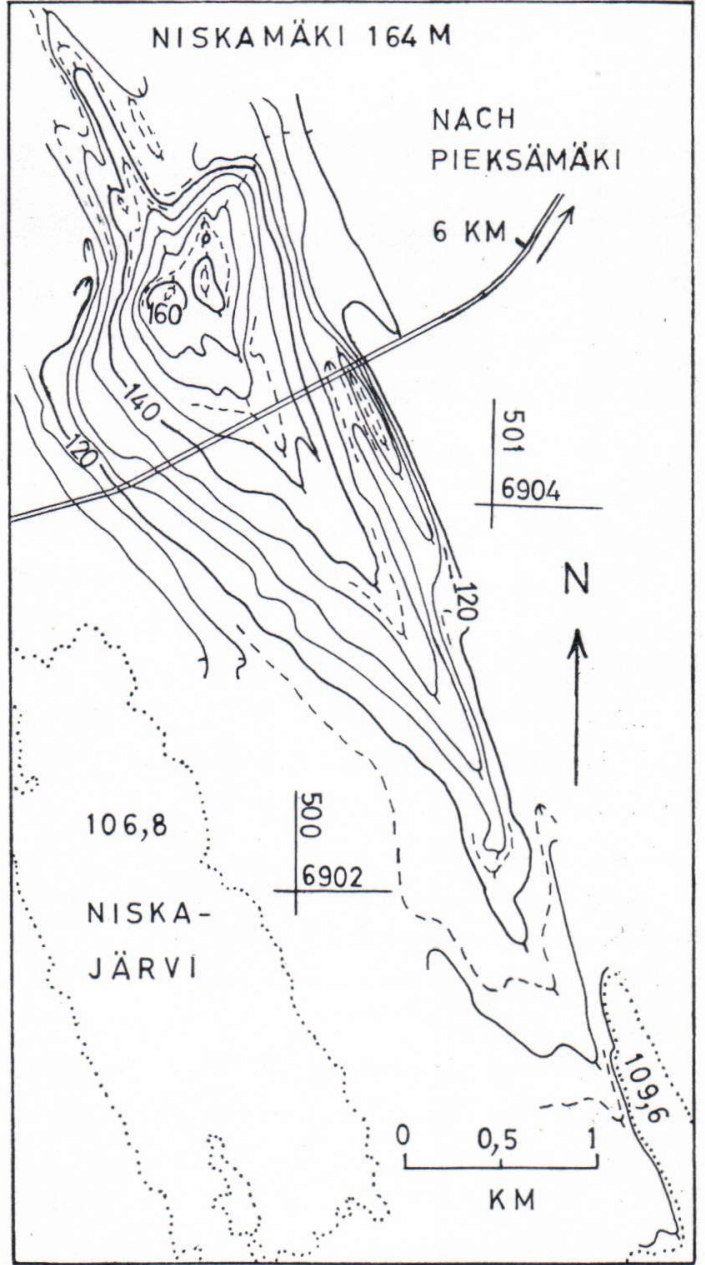

Abb. 6. Der $7 \mathrm{~km}$ lange und $60 \mathrm{~m}$ hohe Niskamäki (164 m ü.d.M.) ist der längste Drumlin des Untersuchungsgebiets und liegt etwa $6 \mathrm{~km}$ SW von Pieksämäki. Verkleinerte Kopie aus den topographischen

Karten, $1: 10$ 000, Nr. $321411 \mathrm{C}$ und 323202 A.

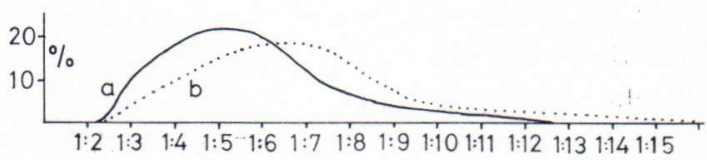

Abb 7. Das Verhältnis zwischen Breite und Länge der Drumlins ist im Nordteil (a) des Untersuchungsgebiets geringer als im Süden. Die meisten Drumlins erreichen das 4- bis 6 fache der Breite. Die Breiten- und Längenmessungen sind aus den topographischen Karten und den Luftaufnahmen. 
ist, womit er den "Selkä-Typ» meint. Alden (1905 S. 19, Fig. 3-6) hat verschiedene Drumlinformen gezeichnet. Fairchild (1907 S. 405, 422 - 423 und Plates 6, 7, 11-14) teilt die Drumlins nach Höhe, Länge und Breite in neun Formtypen ein. Wenn die Eisbewegung konstant bleibt, sind die Drumlins »elongated ridges», aber wenn die Bewegungsrichtung variiert, sind die Drumlins ovalförmig (Wright 1912 S. 158). In Ebers (1926 S. 176-177) Klassifikazion sind die Rückentypen in »mamillary $(<1: 3)$, lenticular (1:3-1:5) und elongated ridges» $(>1: 5)$ eingeteilt. Tanner (1938 S. 489) hat eine Typenserie von »elongated ridges» (der häufigste Typ), »elliptical und mamillary hills» entwickelt.

Gillberg (1955 S. 490, 510-515) legt eine Typenserie der Drumlins in seiner Untersuchung über Schweden vor. Er beginnt mit Stossseitendrumlins, die markierte, aber nicht begrenzte Moränenhügel sind und meistens auf den oberen Teilen von Talflanken vorkommen (Gillberg 1955 S. 510). Die Druml(in)oiden besitzen eine deutliche Form und sind gut begrenzte Hügel (vgl. auch Ebers 1926 S. 232, 1937 S. 233, Fairchild 1929 und Dean 1953 S. 19-23). Die eigentlichen Drumlins bilden die letzte Form der Typenserie (Gillberg 1955 S. 513). Sie liegen meistens auf Plateauflächen (vgl. Högbom 1905 S. 193, Ebers 1926 S. 163, Fairchild 1929 S. 12 und Schmidle 1932) und den proximalen Seiten von Plateaus und sind auf ihren ansteigenden Stossseiten akkumuliert (vgl. Ebers 1926 S. 170, 1937 S. 226, Fairchild 1929 S. 13 und Björnsson 1953 S. 121-123). Neulich haben Chorley (1959 S. 339 - 344) und Reed, etc. (1962 S. 200210) mittels der Luftaufnahmen und topographischer Karten Grösse und Abstand der Drumlins gemessen und daraus geometrische Formenmodelle für Drumlins entwickelt.

Wir finden unter den Drumlins Mittelfinnlands durcheinander verschiedene Formtypen von linearen Rücken, die Chamberlins (1883 S. 306, 1894 S. 522) »linear oder elongated ridges» entsprechen. Die meisten Drumlins in Finnland gehören zum gleichen Typ (vgl. Hän-

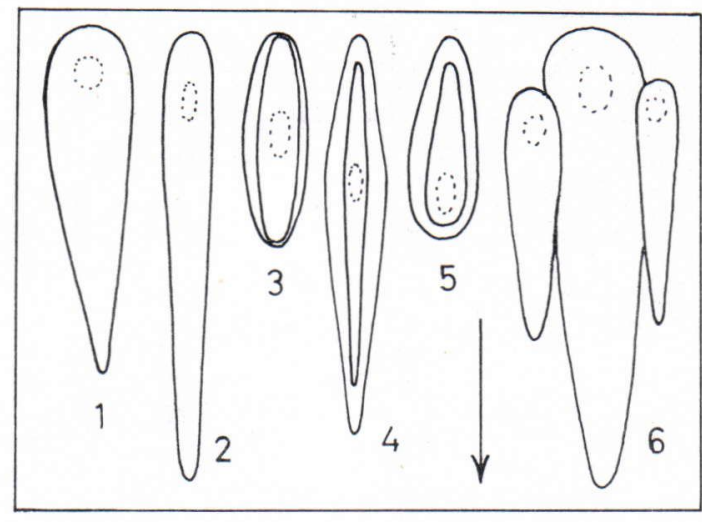

Abb. 8. Schematische Skizze von den im Untersuchungsgebiet vorwiegend auftretenden Drumlinstypen, die verschiedene Variationen der »elongated ridges» darstellen. Die häufigsten Formen sind 1, 2 und 4. Pfeil $=$ Bewegungsrichtung des Inlandeises. Punktiert $=$ höchste Stelle des Rückens.

ninen 1915 S. 13, Tolvanen 1917 S. 104, Tanner 1938 S. 489, Korpela 1954 S. 37, Kurimo 1964 und Aartolahti 1966 S. 44, 1968 S. 23).

Die in Mittelfinnland von Verfasser untersuchten Drumlins können in vier Hauptformtypen eingeteilt werden. Abb. 8 zeigt den Typ 1 mit dem Untertyp, Typ. 2, die häufigste und grösste Form, deren lange, hohe und grossmittelgrosse »lee-side» Rücken vom »Selkä-Typ» besonders im Nordteil des Untersuchungsgebiets allgemein vorkommen (vgl. Abb. 6 und 13). Typ 2 ist am häufigsten im ganzen Mittel- und Südteil des Untersuchungsgebiets, seine Rücken sind lang und meistens mittelgross (Abb. 9 und 10). Typ 3, selten, umfasst kleine und mittelgrosse Rücken (vgl. Abb. 10). Die Drumlins von Typ 4 treten im Mittel- und Südostteil des Gebiets häufig auf. Es sind lange und niedrige, kleine und mittelgrosse Rücken. Sehr schmale Drumlins von diesem Typ können $z u$ den »flutings» gezählt werden. Die kleinen Rücken vom Typ 5 sind in Mittelfinnland selten. Typ 6 stellt Typ 1 mit kleinen Seitendrumlins dar, die besonders im Nordteil des Forschungsgebiets hier und da anzutreffen sind (z.B. Abb. 9).

Die Längsform der Rücken ist bei langen und niedrigen Drumlins meistens annähernd sym- 


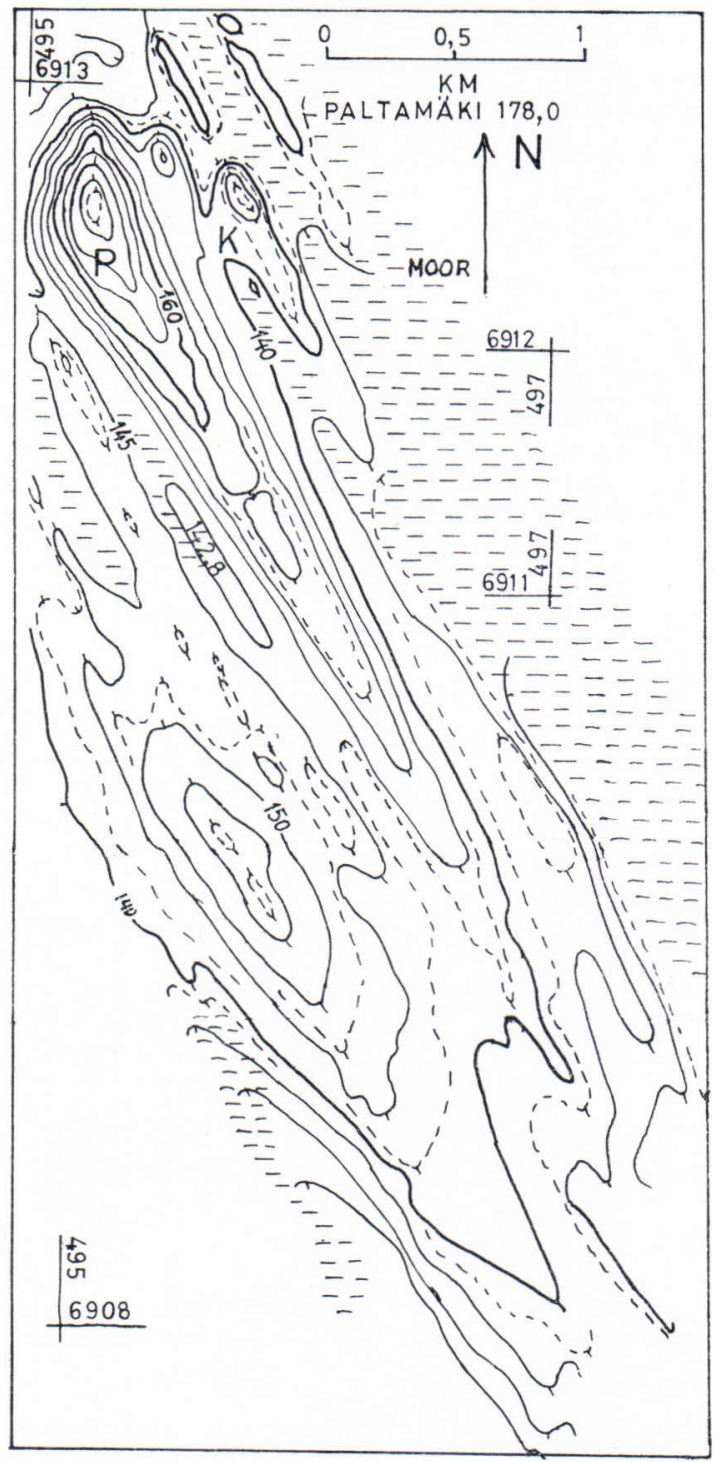

Abb. 9. Der $5 \mathrm{~km}$ lange und $50 \mathrm{~m}$ hohe, typisch ausgebildete Drumlin Paltamäki (P) in Paltanen mit mehreren Seitendrumlins, u.a. dem Kleinen Paltamäki (K), liegt von ebenen Moorsenken umgeben. Verkleinerte Kopie aus den topographischen Karten, 1 : 10 000, Nr. 321412 C und $321411 \mathrm{D}$.

metrisch. Die grossen Rücken sind wegen ihres Felskerns, der fast immer in ihrem proximalen Teil eingebettet liegt, an dieser Stelle am höchsten. (Abb. 11 und 12). Die Querschnitte sind gewöhnlich beinahe symmetrisch. Auch ander-

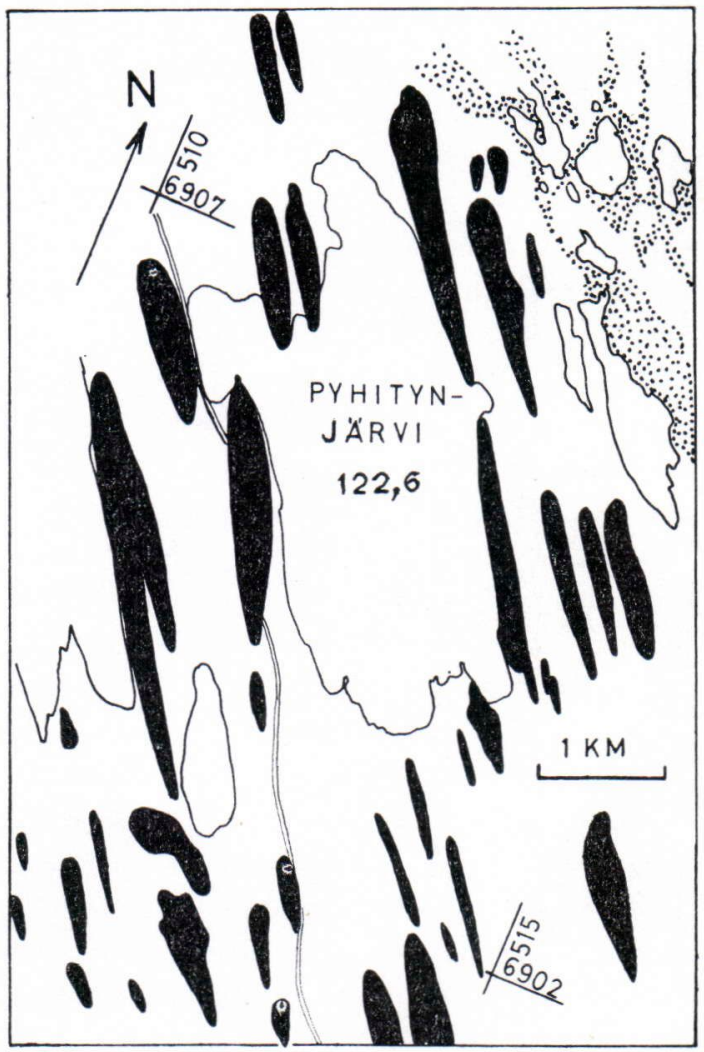

Abb. 10. Karte von der Umgebung des Sees Pyhitty $5 \mathrm{~km}$ SO von Pieksämäki auf der Wasserscheide, ausgezeichnete Drumlinlandschaft mit langgezogenen Moränenrücken, in der die Geländestreifung mit auffallender Deutlichkeit hervortritt. Punktierte Gebiete sind Osbildungen. Verkleinerte Kopie aus den topographischen Karten, 1:10 000, Nr. 323205 A und B.

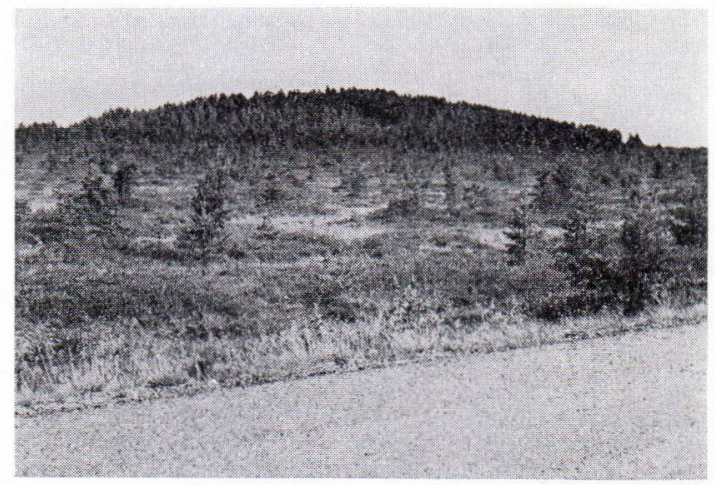

Abb. 11. Das über 40 hohe Proximalende des Drumlins Uhomäki (160 m ü.d.M.) in Pieksämäki aus etwa $1 \mathrm{~km}$ Entfernung von $\mathrm{N}$ her gesehen. Vgl. Abb. 5 und 12. Photo Glückert 1. 8. 1970. 


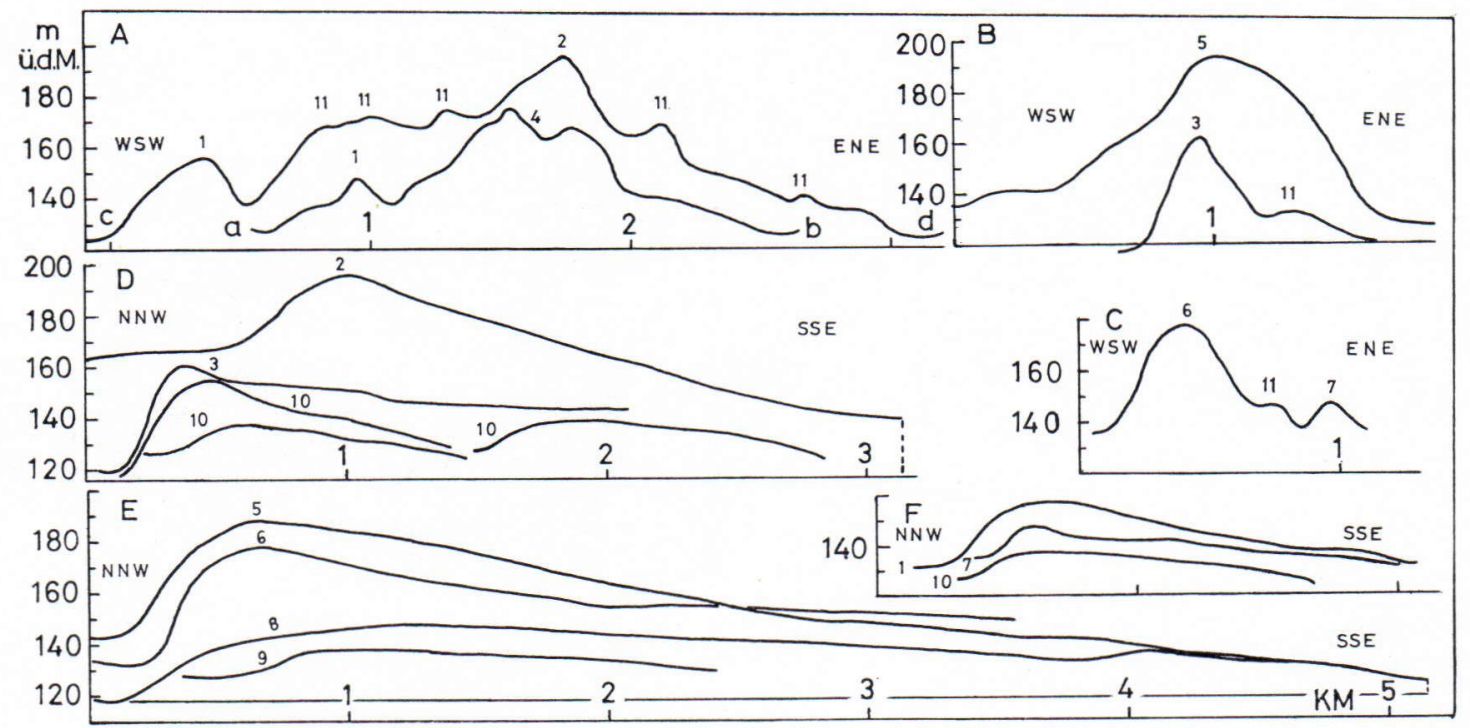

Abb. 12. Quer- (A, B, C) und Längsprofile (D, E, F) der Drumlins in der Gegend von Pieksämäki. A zwei Querprofile des Drumlinschildes Seppälänmäki-Pirttimäki 3 km SW von Pieksämäki (s. Abb. 14). 1. Karjalankylänmäki, 2. Kokkomäki, Kokkomäki-Pirttimäki, 3. Uhomäki, 4. Seppälänmäki, 5. Liperomäki, 6. Paltamäki, 7. Kleiner Paltamäki, 8. Vilhulanmäki, 9. Soiselkä, 10. Drumlins in der Gegend d:s Sees Pyhitty, 11. Kleine Seitendrumlins. Die Profile sind aus den topographischen Karten, 1: 10 000, Nr. 321412 C, 323202 B und D, 3232 03 A, B und $\mathrm{D}$ und $323205 \mathrm{~A}$ und $\mathrm{B}$, gemessen worden.

orts sind die Seitenhänge symmetrisch und das Längsprofil unsymmetrisch (vgl. z.B. Alden 1905 S. 18-19 und Fig. 7., Plates I, III, IV und V, Sahlström 1909 S. 7, Ebers 1926 S. 178-182, 1937 S. 208, Fairchild 1929 S. 15, Schmidle 1932, Gillberg 1955 S. 514-515 und Heidenreich 1964 S. 103-104).

Das Gefälle der Seitenhänge beträgt durchschnittlich etwa $4-20^{\circ}$. Das Proximalende fällt oft um $10-20^{\circ} \mathrm{ab}$, wenn es einen Felskern enthält, und die Distalfläche neigt sich um $1-5^{\circ}$.

Alden (1905 S. 22-25) hat in Amerika zweiund dreiteilige und -scheitelige Drumlins untersucht. In Mittelfinnland sind Drumlins, bei denen Parallelrücken an ihrem Distalende miteinander verwachsen sind, nicht anzutreffen. SO vom Naarajärvi erstreckt sich ein Drumlin (Kortemäki), bei dem zwei parallele Rücken ein gemeinsames Proximalende aufweisen. Ein anderer derartiger Zwillingsdrumlin kommt im Gebiet des Pyhitynjärvi vor (Abb. 1 und 10).
Kleine und niedrige Seitendrumlins schliessen sich oft dem proximalen Ende der grossen Hauptrücken an, wie z.B. dem Paltamäki, Liperomäki und Seppälänmäki, Landgemeinde Pieksämäki (vgl. Abb. 13 und 14; auch Alden 1905 S. 22-25 und Fairchild 1907). Solche Hauptdrumlins mit etwa zwei Nebenrücken sind nicht immer als Drumlinschilde anzusehen. Auch in der Mitte der Drumlinschilde besteht im allgemeinen ein grösserer Mitteldrumlin als die übrigen, der mit seinen kleineren Begleitern genetisch und formal unterschiedliche Drumlinierungsstufen darstellt.

Eine andere gut ausgebildete Drumlingruppe, im Gebiet Seppälänmäki-Pirttimäki, SO vom Vangasjärvi in Pieksämäki, vertritt einen typischen Drumlinschild, wie er auch in anderen Drumlinfeldern Finnlands anzutreffen ist, z.B. in Kuusamo (Hänninen 1915 S. 15, 51), SüdOstbottnien (Tolvanen 1917 S. 103-104) und Häme (Aartolahti 1966 S. 44). Den Sockel vom 


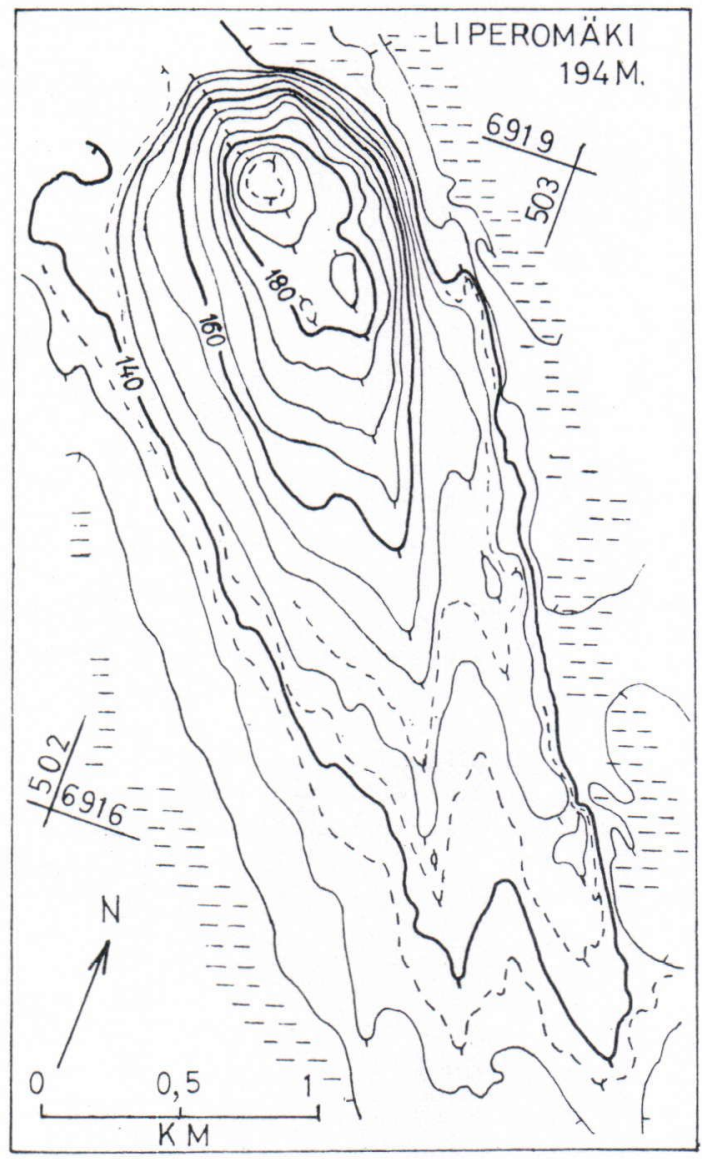

Abb. 13. Der über $4 \mathrm{~km}$ lange und $70 \mathrm{~m}$ hohe Drumlin Liperomäki nördlich von Pieksämäki ist einer der höchsten Moränenrücken des Untersuchungsgebiets. Verkleinerte Kopie aus der topographischen Karte, $1: 10000, \mathrm{Nr} .323203$ B.

Drumlinschild des Seppälänmäki bildet ein mehrteiliger Felshügel, auf dessen Scheitel und Distalseite sich das Moränenmaterial in vielen nebenund hintereinander gelegenen Rücken angeordnet hat (vgl. Korpela 1954 S. 38). Oft haben sich die Drumlingruppe und auch ein Hauptdrumlin mit seinen Nebenrücken im Untersuchungsgebiet auf einer flach gewölbten, schildförmigen Unterlage niedergelassen, wie z.B. die Rückenreihe zwischen Paltanen und Niskamäki. (Abb. 12 A, 14 und 15).

Im Drumlinfeld Mittelfinnlands erscheinen die Rücken in grossen Scharen ohne deutliche

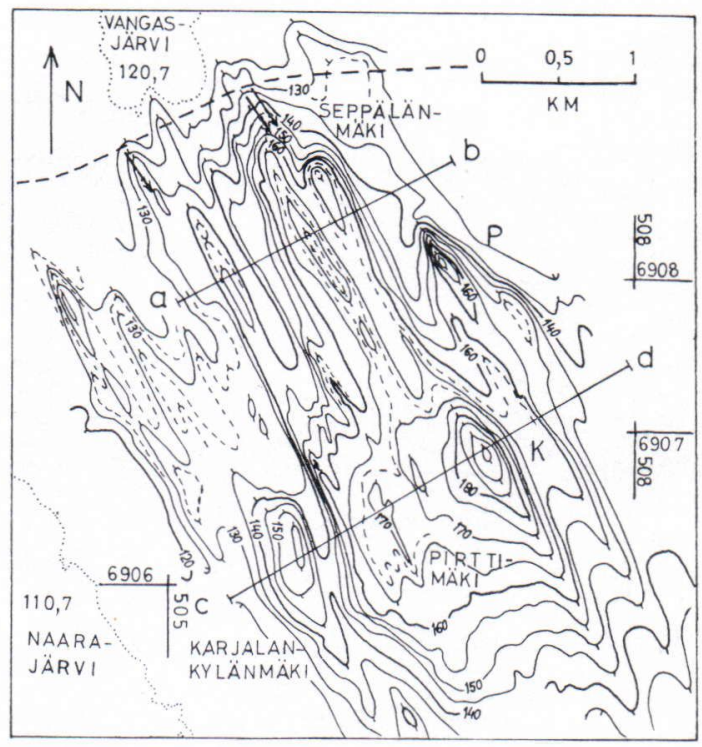

Abb. 14. Der Drumlinschild Seppälänmäki-Pirttimäki zwischen Pieksämäki und Naarajärvi mit zwei Querprofilen (s. Abb. 12). $\mathrm{P}=$ Pappilankallio, $\mathrm{K}=\mathrm{Kokkomäki}$, Pfeile $=$ Schrammen, $320-325^{\circ}$. Verkleinerte Kopie aus den topographischen Karten, 1:10 000, Nr. $323202 \mathrm{~B}$ und D.

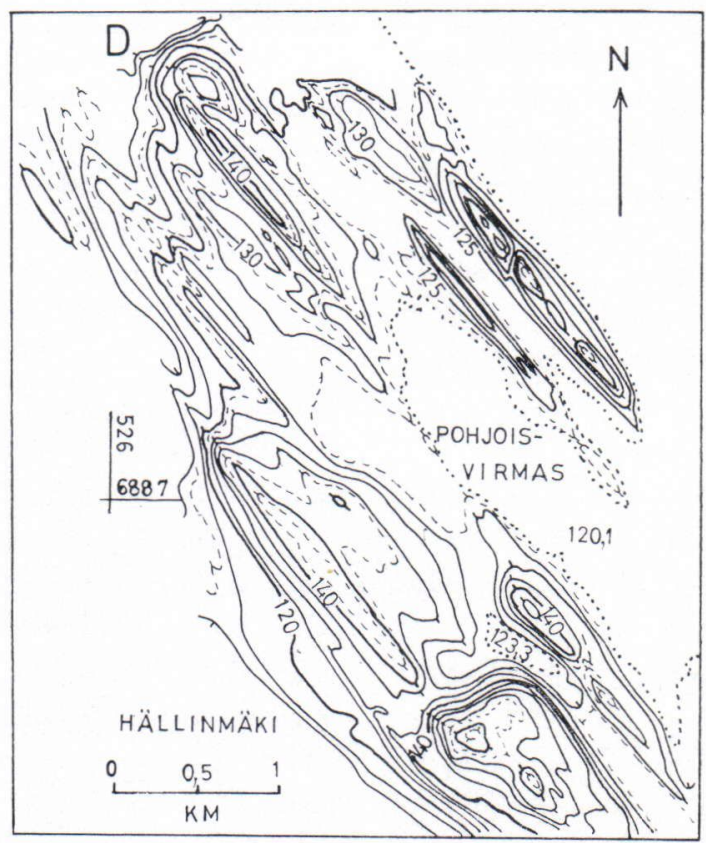

Abb. 15. Drumlinlandschaft in der Gegend von Hällinmäki am W-Ufer des Pohjois-Virmas (120,1 m ü.d.M.) $\mathrm{SE}$ von Virtasalmi mit einem Drumlinschild (D) im Nordteil der Karte. Verkleinerte Kopie aus der topographischen Karte, 1 : 10 000, Nr. 323109 D. 
Grenzen. Die extremen Formen treten nicht zusammen auf, sondern die Drumlins einer und derselben Gegend sind einander recht ähnlich, besonders hinsichtlich der Verhältniszahlen von Länge und Breite.

Die Ausrichtung der Drumlins geht aus der Übersichtskarte des Untersuchungsgebiets deutlich hervor (Abb. 1). Die Drumlinrücken haben sich, vom NW-Winkel des Gebiets aus betrachtet, strahlenförmig ausgebreitet. Im Felde erscheint eine ähnliche fächerförmige Struktur wie in einigen ausgedehnten Drumlinfeldern in anderen Inlandeisgebieten (vgl. Früh 1896, Fairchild 1907, Flint 1957 S. 68 und Wright 1957 S. 21). Eine gleiche fächerförmige Ausstrahlung lässt sich auch in den Richtungen der Schrammenbeobachtungen in den verschiedenen Teilen des Untersuchungsgebiets erkennen.

Die Orientierung verbindet sich also als wesentlicher Faktor mit den Bewegungen der Vereisung (vgl. z.B. Hänninen 1915 und Aartolahti 1966 S. 45). Brenner (1944 S. 19, 38) hat dargelegt, dass sich der Rand des Inlandeises über Binnenfinnland hinweg von NW nach SO vorgeschoben und in drei riesige Zungen verzweigt habe (vgl. auch Brander 1934). Die Landeislobe von Savo, die zwischen zwei Nachbarloben geklemmt war, hat sich über die Gegend von Pieksämäki nach SO bewegt, und die Hauptbewegungsrichtung liegt auf der Linie Pieksämäki-Lappeenranta.

\section{Aufbau und Material}

In Schnitten von Strassenanlagen und Eisenbahnen im Untersuchungsgebiet haben Material und Innenbau der Drumlins erforscht werden können. Einige Moränenproben sind entnommen und analysiert worden. Die Drumlins bestehen aus hartgepackter, lehmartiger Grundmoräne, deren Material aus heterogenem Schluff, Feinsand und Sand mit unterschiedlich grossen, kantigen, an den Ecken abgerundeten Steinen besteht.

Die primäre dichte, allgemein ungeschichtete Struktur der Grundmoräne hat sich beinahe bis
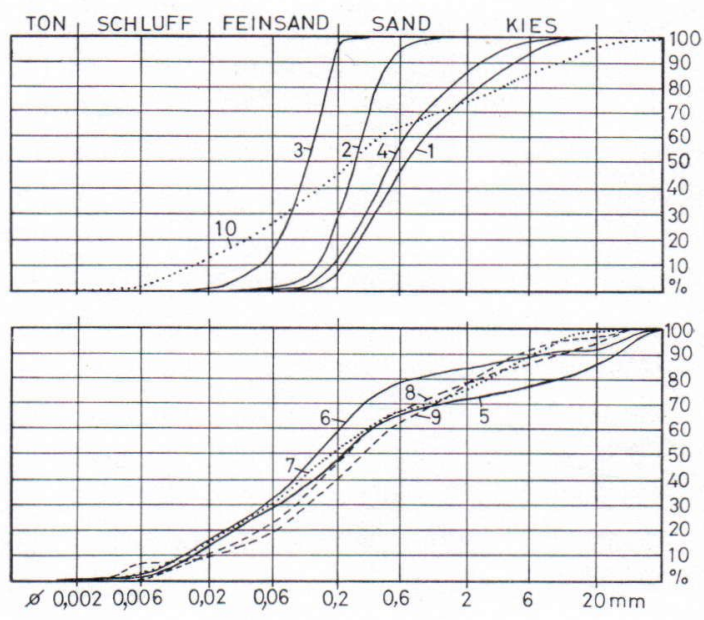

Abb. 16. Kornverteilungskurven für glazifluvilales Material (1-4) und Moräne (5-10) aus der Gegend von Pieksämäki. Osmaterial: 1. Naarajärvi, Tiefe $3 \mathrm{~m}, 123 \mathrm{~m}$ ü.d.M., Kordinaten 69 0938/5 0143, 2. Naarajärvi, $2,5 \mathrm{~m}, 116 \mathrm{~m}, 690832 / 50217,3$. Naarajärvi, $1 \mathrm{~m} ; 117 \mathrm{~m}$, $690798 / 50240,4$. SO von Naarajärvi, drumlinartiger Osrücken, $1 \mathrm{~m}, 121 \mathrm{~m}, 69$ 0358/5 0346, Moräne aus den Drumlins: 5. Niskamäki, 2 m, 135 m, 69 0468/5 0026, 6. Soiselkä, 2 m, 131 m, 69 0984/5 0682, 7. Karjankylänmäki, 1,5 m, 136 m, $690857 / 5$ 0484, 8. Liperomäki, 1 m, 166 m, 69 1797/5 0198, 9. Pirttimäki, 2 m, 165 m, 69 0697/5 0737, 10. Paltamäki, $1 \mathrm{~m}, 152 \mathrm{~m}, 691274 / 4$ 9533.

zur Oberfläche unverändert erhalten. An der Oberfläche lagert eine $0,2-1 \mathrm{~m}$ mächtige sandige, lockere Schicht, die in erster Linie den nach dem Schwinden des Eises veränderten Oberflächenteil der Grundmoräne darstellen dürfte. Stellenweise ist die Moräne steinhart und beinahe blockfrei, hier und da ist der Oberflächenteil der Massen sehr blockreich, und zwar blockreicher als die tieferen Ablagerungen (vgl. Fairchild 1929, Björnsson 1953 S. 108) (Abb. 16).

In einigen neuen Drumlinschnitten hat eine undeutliche polsterförmige Schichtung wahrgenommen werden können. Eine derartige Lager- und Lamellenstruktur ist in der Moräne als Ergebnis innerer Bewegung der Vereisung entstanden (z.B. Virkkala 1948 S. 27-31, Korpela 1954, Aartolahti 1966 S. 45 und 1968 S. 25). Erstere dürfte die in stärkeren Schichten erfolgte Bewegung des Eises widerspiegeln, letztere die im Eise geschehene durchgehende Bewegung. 
Echte Felsdrumlins gibt es im Untersuchungsgebiet nicht (vgl. z.B. Fairchild 1907 S. 422, 1929 S. 25, 29, Ebers 1926 S. 227, Gillberg 1955 S. 490, Flint 1957 S. 68, Svensson und Frisén 1964 S. 19-30, Aartolahti 1968 S. 25-26, Virkkala 1969 S. 11, 60 und Lundqvist 1970 S. 317). Im allgemeinen bildet den Kern eines typischen Drumlins ein Fels (vgl. Högbom 1905 S. 176 und Ebers 1926 S. 223), aber lange und niedrige Rücken können ausschliesslich aus Moräne bestehen (vgl. Ebers 1926 S. 254, 1937 S. 218, Fairchild 1929 S. 28, Virkkala 1960 S. 19-20 und Lundqvist 1970 S. 317). Tanner (1938 S. 487) teilt die Rücken in Grundmoränen- und Kerndrumlins mit allen Übergangsstadien ein.

Einige mit Kiefern bewaldete, osähnliche, lange und schmale, wie Drumlins gestaltete und ausgerichtete Rücken vertreten eine Zwischenform von Os und Drumlin. Verhältnismässig sortiertes und ausgewaschenes Material ist stellenweise von einer Moränendecke umgeben. Derartige Rücken sind u.a. SW-und S-seits des Sees Naarajärvi in der Nähe von Pieksämäki anzutreffen, und diese Bildungen sind in der vorliegenden Untersuchung als Ose angesehen worden. Upham (1889 S. 228-242), Tanner (1915), Ahlmann (1917), Virkkala (1948 S. 26, 1951 S. 37-38), Jaatinen (1952 S. 62, 1960 S. 14-17), Kupsch (1955 S. 327), Flint (1957 S. 68), Lemke (1958 S. 281-282), Hausen (1964 S. 138), Wisniewski (1965 S. 174-178) und Aartolahti (1968 S. 25) haben andernorts innerhalb von Drumlins in Moräne Schichten, Linsen und Polster sortierten Materials beobachtet.

Die Höchste Grenze an der Wasserdecke der Ostsee hat in der Gegend von Pieksämäki bis zu einer Höhe von etwa 130 m ü.d.M. gereicht (Ramsay 1931 S. 97). Nach Ramsay ist die Höchste Grenze beinahe überall durch Bodenkultur verwischt. Doch lässt sie sich durch Veränderungen im Gefälle des Terrains und hier und da ebenfalls durch wohlausgeprägte Uferbildungen, z.B. in der Nähe von Uhomäki, Pieksämäki, im Hang von Drumlins und anderen Hügeln immerhin noch gut erkennen.
Die ursprünglichen Oberflächenformen der Drumlins sind in den unter $130 \mathrm{~m}$ liegenden Gegenden durch Einwirkung nacheiszeitlicher Brandung modifiziert worden. Brandungen haben die bis in $130 \mathrm{~m}$ Höhe liegenden Abschnitte der Drumlinsflanken abgewaschen. Auf die untersten Teile der Rücken und in die trogartigen Senken sind oft Sand, Feinsand und Schluff auf die Grundmoräne niedergeschwemmt worden. Diese Sedimente bilden den unmittelbaren Untergrund der Moore dieser Senken. Lehmige Bestandteile gibt es in ziemlich geringen Mengen, weshalb die Gegend unter Mangel an für Ackerbau geeignetem Boden leidet (vgl. Högbom 1905 S. 180). Trotzdem sind die meisten Scheitelteile der grossen Drumlins bebaut (vgl. z.B. Sauramo 1928 S. 175 und Björnsson 1953).

\section{Entstehung der Drumlins}

Noch hat man keine Theorie entwickelt, die das Entstehen der Drumlins erklären könnte. In den zusammenfassenden Arbeiten über Drumlins hat besonders Edith Ebers (1926, 1937) Form und Entstehung dieser Bildungen dargelegt. Die Ergebnisse jener eingehenden Untersuchung stimmen in ihren Hauptzügen auch weiterhin mit den anerkannten Auffassungen überein.

Allgemein sind Lage und Vorkommen der Drumlins von der absoluten Höhe des Gebiets und von der Topographie des Geländes abhängig. Die Drumlins von Mittelfinnland liegen hauptsächlich in ebenem Wasserscheidegebiet in rd. 100-130 m ü.d.M., d. h. in der Zone des höchsten Ufers der Ostsee und etwas darunter. Auch in Ostfinnland und Schweden liegen die am besten entwickelten Drumlingelände in ebenen, aber niedrigen Gegenden etwas unter dem höchsten Ufer. Auf ebenen Böden hat sich das Eis gleichmässig bewegt, und das von ihm mitgeführte Material hat sich durch ein entgegenstehendes Hindernis am leichtesten zu einem mit der Eisbewegung gleichgerichteten Rücken aufschütten können. In Gebieten mit unebenem Relief sind im allgemeinen keine Drumlins 
anzutreffen, da sich die Bewegung des Eises seinem Untergrund mehr angepasst hat (vgl. Chamberlin 1883 S. 306, Högbom 1905, Fairchild 1907, Virkkala 1948 S. 26, Hoppe 1959 S. 204, Aartolahti 1968 S. 26-27 und Lundqvist 1970 S. 317). Zu gleichmässig gerade weiterführender Bewegungsrichtung des Eises hat das Zusammenfallen der Schieferung des Felsgrundes mit ihr beigetragen (z.B. Aartolahti 1966 S. 47).

Nach Ansicht des Verfasser ist das Drumlinfeld im Gebiet Pieksämäki-Haukivuori teils sowohl zur Zeit der eigentlichen Vereisung als auch teils während der Zeit des letzten Zurückziehens unter dem Gletscher aus Moränenmaterial der unteren Eismassen als Ergebnis von Erosion und Akkumulation in mehreren verschiedenen Phasen entstanden. Auf der höher als ihre Umgebung gelegenen, ebenen WasserscheideHochfläche Mittelfinnlands führte die Vereisungszunge sanft bergauf, wodurch sich vorwiegend die Bewegung der unteren Eispartien verlangsamte. Dies bewirkte ein Aufschütten von Moräne phasenweise um die der Bewegung entgegenstehenden Felskerne, die sich dadurch alimählich vollends in Moränenmassen einbetteten. Die bereits akkumulierte, durch das Eis dichtgepresste Grundmoräne wurde zugleich der Erosion der sich bewegenden Eismassen ausgesetzt. Abtragung hat sich auch während des Zurückweichens des Inlandeises vollzogen. Durch Horizontaldruck hat die eisbedingte Akkumulations-Erosionskette den Rücken zugleich ihre längliche Stromlinienform verliehen.

Die Erosions- und Akkumulationstätigkeit des Inlandeises war abhängig von seiner Mächtigkeit, Bewegungsgeschwindigkeit, Moränenmenge und der Topographie seines Untergrundes. Offenbar verminderte sich die Geschwindigkeit des Eises, wenn sich die von ihm mitgerissene Moräne vermehrte und wenn das Relief des Untergrundes zu steigen begann oder etwas unebener wurde. In der an der Basis der Vereisung angesammelten Moräne war die Reibung stärker als weiter aufwärts im Eise. Somit hafteten die reichlich Moräne führenden basalen Partien am leichtesten an ihrer Unterlage, indem das Verfrachtungsvermögen des Eises während seiner verlangsamenden Bewegung schwächer wurde. Während sich ein Teil der Moräne fest an seine Unterlage presste, bewegte sich darüber noch das weniger Moräne enthaltende Eis und vollendete zugleich Längsrichtung und Form der Moränenhügel. Die in Phasen vor sich gegangene Akkumulation, die Reibung zwischen Felsgrund, Moräne und Eis schufen in den Drumlins die Polster- und Lamellenstruktur, die bezeugt, dass die Grundschichten der Moräne schon fest mit ihrem Untergrund verkittet waren, als sich die oberen Teile noch bewegten (vgl. Virkkala 1948 S. $27-31$ und Korpela 1954 S. 41-45).

Über die Entstehung der Drumlins sind Auffassungen von zweierlei Typ vorherrschend gewesen. Nach dem einen haben sich die Drumlins unter der sich bewegenden Eismasse hauptsächlich als Ergebnis der Akkumulationstätigkeit des Eises gebildet, nach dem anderen hingegen ist ihre Entstehung überwiegend erosiv gewesen. Die meisten Quartärgeologen sind der Ansicht, dass die Drumlins zum grössten Teil Ablagerungsformen von Grundmoräne sind (z.B. Högbom 1905 S. 176-184 und Lundqvist 1970 S. 325 - 326), die sich beim Vorstoss oder Rückzug des Inlandeises herausgestaltet haben (vgl. z.B. Fairchild 1907, 1929 Ebers 1926, 1937 und Flint 1957).

Nach der allgemeinen Auffassung sind die Drumlins in der Anfangsphase subglazialer Akkumulation durch die Bewegung des Eises entstanden (Ebers 1937), der die erosive Tätigkeit des Eises folgte (Fairchild 1907, Ebers 1926). Man hat die Drumlins als submarginale Gebilde angesehen (Taylor 1906, Ebers 1926, Hoppe 1951), die in Niederungs-, Becken- und auch Hochflächengebieten vorkommen (vgl. Lundqvist 1970 S. 325). Die Mächtigkeit der Massen steigert sich in Drumlingebieten (Hoppe 1951), woraus man geschlossen hat, dass sie in Basalteilen des Eises durch Moränensättigung entstanden seien (Lundqvist 1970 S. 324-325). 
Nach Högbom (1905 S. 176-184) bewirkt das Eis erst die Erosionsformen des Felsgrundes und geht danach zu der ablagernden, drumlinbildenden Bearbeitung über. Die Felskerne verursachen die Entstehung der Drumlins, und die Schichtstruktur ist das Ergebnis von Eisgewicht und in Phasen erfolgender Ablagerung von Material. Nach Alden (1905 S. 43, 1911) ist die endgültige Form der Drumlins während des letzten Vorstosses des Inlandeises in längsgerichteten Gletscherspalten entstanden.

Nach Fairchilds (1907, 1929) Auffassung hat sich während des Eisvorstosses unter Eisdruck Grundmoräne um einen Primärbuckel akkumuliert (vgl. Högbom 1905 S. 195, Ebers 1926 S. 254, 1937 S. 218 und Björnsson 1953). Beim Hinweggleiten des Eises über den Buckel hat sich der Rücken ausgerichtet, seine Stromlinienform angenommen (vgl. Högbom 1905 S. 192 und Ebers 1937 S. 236) und das geringste Hindernis gebildet sowie einen Gleichgewichtszustand zwischen pressenden und abtragenden Kräften im Eise hergestellt (vgl. Slater 1929 S. 19),

Der Horizontaldruck der Vereisung ist besonders dann, wenn die Akkumulation bergauf vor sich gegangen ist, der Hauptfaktor gewesen (vgl. Alden 1911, Fairchild 1929, Slater 1929). Vollzog sich in den Randteilen des Eises ein Abschmelzen, so verringerte sich sein Druck so sehr, dass es sich unter dem Druck des mächtigen Mittelteils seitwärts zu schieben begann, wobei sich der Gletscher in seiner Ganzheit vorwärts bewegte. Hier dürfte sich der Schlüssel zur Entstehung der Drumlins finden (vgl. Fairchild 1929 S. 29-30 und Gillberg 1955 S. 517).

Nach Ebers (1937 S. 231) und Tanner (1938 S. 492) gelangen in den Drumlins die erodierende und die akkumulierende Tätigkeit des Eises gemeinsam zur Wirkung. Dabei sind die Drumlins im stark fliessenden Gletscher subglazial zustandegekommen (vgl. auch z.B. v. Klebelsberg 1948 S. 280). Über die Notwendigkeit des Vorhandenseins von Kernen besteht noch keine
Einigkeit. Nach Tanner (1938 S. 492) sind die Kern- und Grundmoränendrumlins aus Moräne subglazial geformte Stromlinienkörper, die den geringsten Widerstand gegen die Überfahrungsbewegung des Eises leisten.

Die langen, schmalen »linear ridges» sind nach Hoppe und Schütt (1953) aus in Eistunnel gepresstem Moränenmaterial entstanden. Gemäss Björnsson (1953 S. 122-124) sind die Drumlins, die er in Proximal- und Distaldrumlins einteilt, unter der vordringenden Vereisung, unter starkem Druck nahe dem Eisrand entstanden. Nach Gravenors (1953 S. 679) Auffassung schüttete sich bei Stillstand des Gletschers Moräne und sortiertes Material vor dem Eise auf. Danach drang über diese hinweg das Eis vor und gestaltete aus dem Material drumlinförmige Rücken. Gemäss der Theorie Lemkes (1958 S. 281-282) schüttete das Eis zur Bedekkung dieser aus sortiertem Material bestehenden Rücken über sie einen Moränenmantel aus.

Kupsch (1955 S. 327) schreibt über drumlinisierte Kamesbildungen, deren Kern aus moränebedecktem, sortiertem Material besteht. Nach Vernon (1966 S. 405-408) entstehen Drumlins durch Druckdifferenzen zwischen vorderem und hinterem Teil der Eiszungen, und das Druckschmelzen an der Spitze bewirkt eine schnelle Bewegung des Eises. Smalley und Unwin (1968 S. 377-381) haben experimentelle Untersuchungen über die Entstehung der Drumlins angestellt. Nach ihnen ist das Modell des Entstehungsmechanismus davon abhängig, dass der Gletscher ständig durch eine sich deformierende Moränenschicht getrennt wird und dass diese Schicht Druckflächen umfasst. Die Entstehung ist in erster Linie das Ergebnis eines Akkumulationsprozesses des Eises.

Lundqvist (1970 S. 325-326) stellt dar, die Drumlins seien als Ergebnis sowohl von Akkumulation als auch Erosion während des Eisrückgangs entstanden. Als das Eis geringmächtiger wurde, verminderte sich sein Vertikaldruck, und der Horizontaldruck wurde zum wichtigsten Urheber der Eisbewegung. 


\section{LITERATURVERZEICHNIS}

Aartolahtr, Torve (1966) Koijärven-Urjalan drumliinikenttä. Summary: The Koijärvi-Urjala Drumlin Field. Terra 78,2, S. 42-50.

- (1968) Die Geomorphologie des Gebiets von Tammela, Südfinnland. Fennia 97,7, S. 1-97.

Ahlmann, H. (1917) Die fenno-skandischen Endmoränenzüge auf und neben dem Billingen in VesterGötland, Schweden. Zeitschr. für Gletscherkunde X.

Alden, William C. (1905) The drumlins of southeastern Wisconsin. (Preliminary paper.) U.S. Geol. Survey, Bull. 273, S. $1-46$.

- (1911) Radiation of glacial flow as a factor in drumlin formation. Bulletin of the Geological Society of America, Vol. 22, S. 733-734.

Aronow, SaUl (1959) Drumlins and related streamline features in the Warwick-Tokio area, North Dakota. American Journal of Science, Vol. 257, 3, S. 191-203.

Bergdahl, Arvid (1961) Det glaciala landskapet. Meddelanden från Lunds Universitets Geografiska Institution, Ser. Avhandlingar. N:o 47, S. 201-326.

BJörk, Arne (1959) Drumlins på Kinnekulle. Summary: The drumlins of Kinnekulle. Ymer 1959, 4, Stockholm, S. 241-258.

BJÖRnSSON, Sven (1953) † Drumlinbildningar i SommenÅsundenområdet. Svensk Geografisk Årsbok 1953, Årg. 29, Lund. Summary: Drumlins in the Region of Sommen and Åsunden, South-Eeast Sweden. The Swedish Geographical Yearbook 1953 (29), S. 104126.

Brander, G. (1934) Suomen geologinen yleiskartta, Lehti C3, Kuopio. Maalajikartan selitys. Suomen geologinen toimikunta. Geologiska Kommissionen i Finland, S. $1-67$.

Brenner, Thord (1944) Finlands åsars vittnesbörd om ytgestaltningen hos landisen. Zusammenfassung: Die Osen Finnlands als Zeugen der Oberflächengestaltung des Landeises, Fennia 68, 4, S. 1-39.

Chamberlin, Thomas C. (1883) Preliminary Paper on the terminal moraine of the Second glacial epoch. Third annual report of the U.S. Geol. Survey by I. W. Powell, Washington, S. 291-406.

- (1894) Proposed Genetic Classification of pleistocene glacial Formations. Journal of Geology 2, V, S. $517-$ 538.

Chorley, Richard J. (1959) The shape of drumlins. Journal of Glaciology, Vol. 3, 25, S. 339-344.

Close, H. M. (1866) Notes on the general glaciation of Ireland. Journal of the Royal Geological Society of Ireland, Vol. 1, 1864-1867, S. 207-242.

Charlesworth, J. K. (1957) The Quaternary Era with special reference to its glaciation. Vol. I. London, S. $1-591$.
Dean, W. G. (1953) The drumlinoid landforms of the „Barren Grounds», N.W.T. The Canadian Geographer 3, 1953, S. 19-30.

Donner, J. J. und West, R. G. (1955) Ett drumlinsfält på ön Skye, Skottland. English summary, J. J. Donner \& R. G. West: A drumlin-field on Skye, Scotland. Terra 67, 2, S. 45-48.

Ebers, Edith (1926) Die bisherigen Ergebnisse der Drumlinforschung. Eine Monographie der Drumlins. Neues Jahrbuch für Mineralogie, Geologie und Paläontologie. Beilagebände 53, Abt. B, S. 153-270.

- (1937) Zur Entstehung der Drumlins als Stromlinienkörper. Zehn weitere Jahre Drumlinforschung (19261936). Neues Jahrbuch für Mineralogie, Geologie und Paläontologie. Beilagebände 78, Abt. B, S. 200-240.

EArdley, A. J. (1965) General College Geology. New York, S. $1-499$.

Embleton, Clifford und King, Cuchlaine, A. M. (1968) Glacial and Periglacial Geomorphology. Edingburgh, S. $1-608$.

Eskola, Pentri (1941) Itä-Karjalan kallioperästä. Referat: Über den Felsgrund Ostkareliens. Terra 53, 4, S. $171-193$.

Fatrchild, Herman L. (1907) Drumlins of central western New York. New York State Museum. Bulletin 111. Geology 13, S. 391-439.

- (1929) New York Drumlins. Proceedings of the Rochester Academy of Science, Vol. 7, 1. Rochester, N.Y., S. $1-37$.

Flint, Richard Foster (1957) Glacial and Pleistocene Geology. New York, S. 1-553.

Frosterus, BenJ. (1902) Geologisk öfversiktskarta öfver Finland. Sektionen C2, S:t Michel. Beskrifning till bergartskartan. Résumé. Geologiska Kommissionen, S. $1-114$.

- (1913) Suomen geologinen yleiskartta. Lehti C2, Mikkeli. Maalajikartan selitys. Geologinen toimisto, S. $1-49$.

FRÜH, J. (1896) Die Drumlinlandschaft mit specieller Berücksichtigung des alpinen Vorlandes. Jahresbericht der St. Gallener naturw. Ges. 1895/96. IX, St. Gallen.

De Geer, G. (1895) Mötet den 7. Mars 1895. Geologiska Föreningen i Stockholm Förhandlingar 17, 3, S. 212 213.

Gillberg, Gunnar (1955) Den glaciala utvecklingen inom Sydsvenska höglandets västra randzon. I. Glacialerosion och moränakkumulation. English summary: Glacial erosion and till accumulation in the Western Marginal Zone of the South-Swedish Highlands. Geologiska Föreningen i Stockholm Förhandlingar 77, H. 4, S. 481-524. 
Gravenor, Conrad P. (1953) The origin of drumlins. American Journal of Science, Vol. 251, 9, S. 674-681.

Gravenor, C. P. und Meneley, W. A. (1958) Glacial flutings in Central and Northern Alberta. American Journal of Science, Vol. 256, 10, S. 715-728.

Hausen, Hans (1964) Geologisk beskrivning över Landskapet Åland. Skrifter utgivna av Ålands Kulturstiftelse IV. Bilaga med XLV planscher, S. 1-196.

Heidenreich, Conrad (1964) Some observations on the shape of drumlins. The Canadian Geographer, Vol. VIII, 2, S. $101-107$.

Hollingworth, G. E. (1931) The glaciation of western Edenside and adjoining areas and the drumlins of Edenside and the Solway basin. Quart. Journ. Geol. Soc. London $87: 2$, S. 281-359.

Holmes, Arthur (1965) Principles of physical geology. London, S. 1-1288.

Hoppe, Gunnar (1951) Drumlins i Nordöstra Norrbotten. Summary: Drumlins in north-east Norrbotten, North Sweden. Geografiska Annaler 33, 3-4, S. $157-165$.

- (1959) Glacial morphology and inland ice recession in Northern Sweden. Geografiska Annaler 41, 4, S. $193-212$.

Hoppe, Gunnar and Schytt, Valter (1953) Some observations on fluted moraine surfaces. Geografiska Annaler 35, 2, S. 105-115.

Hult, R. (1893) Savonselkä i Pieksämäki. Geografiska Föreningens Tidskrift, V, 1, S. 38-40.

HänNINEN, KAARLO (1915) Drumliinimaisemien järvistä ja reiteistä Oulankajoen alueella Kuusamossa, S. 1153. Über die Seen und Seenketten der Drumlinlandschaften im Gebiet des Flusses Oulankajoki, Kirchspiel Kuusamo (Finnland), S. 1-11. Meddelanden av Geografiska Föreningen i Finland. Suomen Maantieteellisen yhdistyksen Julkaisuja XI, 1915-1920.

Нӧявом, А. G. (1905) Studien in nordschwedischen Drumlinlandschaften. Bulletin of the Geol. Inst. of the Univ. of Upsala, Vol VI: Part 2. No. 12, 7, S. $175-199$.

JAAtinen, Strg (1952) Ramsholm - en åländsk drumlin. English summary, Stig Jaatinen: Ramsholm, a drumlin of Alland Island. Terra 64, 2, S. $57-65$.

- (1960) The Glacial Morphology of Aland, with special reference to the Quaternary deposits. Les formes glaciaires de l'ile Åland. Fennia 84, 1, S. 5-38.

v. KlebelsberG, R. (1948) Handbuch der Gletscherkunde und Glazialgeologie. I. Wien, S. 1-403.

Korpela, Kalevi (1954) Pieksämäen vedenjakaja-alueen geomorfologiaa. Unveröffentlichte Laudaturarbeit in der Bibliothek des Geographischen Instituts der Universität Helsinki, S. $1-76$.
Kujansuu, Raimo (1969) Mannerjäätikön liikesuuntien määrääminen ilmakuvista. Ilmakuvien tulkinta. Insinöörijärjestöjen Koulutuskeskus. Ingenjörorganisationernas Skolningscentral, $1-69$, Helsinki, S. $1-8$.

Kupsch, W. O. (1955) Drumlins with jointed boulders near Dollard, Saskatchewan. Bulletin of the Geological Society of America, Vol. 66, 3, S. 327-338.

Kurimo, Heikкr (1964) Etelä-Sallan vedenjakaja-alueen geomorfologiasta. Unveröffentlichte Laudaturarbeit in der Bibliothek des Geographischen Instituts der Universität Helsinki, S. 1-149.

LeIviskä, I. (1907) Über die Oberflächenbildungen Mittel-Ostbottniens und ihre Entstehung. Fennia 25,2 , S. $1-113$.

Lemke, Richard, W. (1958) Narrow linear drumlins near Velva, North Dakota. American Journal of Science, Vol. 256, 4, S. 270-283.

Lundevist, G. (1943) Norrlands jordarter. Sveriges Geologiska Unders. Ser. C., Nr. 457, S. 1-166.

LUNDQVIST, JAN (1970) Studies of drumlin tracts in Central Sweden. Acta Geographica Lodziensia, Nr. 24, S. 317-326.

Mölder, K. und Salmi, Martti (1954) Suomen geologinen yleiskartta. Lehti B3, Vaasa. Maalajikartan selitys. Geologinen tutkimuslaitos, S. 1-109.

Окко, Vегкко (1949) Suomen geologinen yleiskartta. Lehti B4, Kokkola. Maalajikartan selitys. Geologinen tutkimuslaitos, S. 1-108.

- (1964) Maaperä. Suomen geologia. Herausgegeben von Kalervo Rankama. Helsinki, S. 239-332.

Persson, Torsten (1966) Moränmorfologi inom delar av sydsvenska höglandets södra randzon. Summary: Moraine morphology in parts of the southern border of the South-Swedish Highlands. Sveriges Geografiska Årsbok, 1966. Årg. 42. Lund. The Swedish Geographical Yearbook 1966 (42), S. 98-108.

Ramsay, Wilhelm (1931) † Material zur Kenntnis der Spätglazialen Niveauverschiebungen in Finnland. Fennia 54, 3, S. $1-145$.

Renkonen, Olavi (1933) Über die mittlere Höhe Finnlands und seiner geographischen Gebiete. Fennia 58,2 , S. $1-21$.

Reed, Bruce, Galvin, Cyril, J., Jr. und Miller, JoHN, P. (1962) Some aspects of drumlin geometry. American Journal of Science, Vol. 260, 3, S. 200-210.

Repo, Reino (1957) Untersuchungen über die Bewegungen des Inlandeises in Nordkarelien. Bulletin de la Commission géologique de Finlande 179, S. 1-178.

Rosberg, J. E. (1892) Ytbildningar i ryska och finska Karelen med särskild hänsyn till de karelska randmoränerna. (Referat). Oberflächenbildungen im russischen und finnischen Karelen mit besonderer Berücksichtigung der karelischen Randmoränen. Fennia 7, 2, S. $1-128$. 
SAHLSTRÖM, K. E. (1909) Ett drumlinområde i Närke. Sveriges Geologiska Unders. Ser. C., Nr. 222, S. 1-12.

Sauramo, Matti (1928) Jääkaudesta nykyaikaan. Porvoo, S. $1-231$.

Schmidle, W. (1932) Die Drumlinhügel des diluvialen Rheingletschers. Fortschritte Geol. und Paläont., Bd. XI. Berlin, S. $341-375$.

Schytt, Valter (1963) Fluted moraine surfaces. Journal of Glaciology, Vol. 4, 36, S. 825-827.

Sederholm, J. J. (1889) Om istidens bildningar i det inre af Finland. (Auszug). Ueber die Bildungen der Eiszeit im inneren Finnland. Fennia 1, 7, S. $1-52$.

Slater, George (1929) The structure of the drumlins exposed on the south shore of Lake Ontario. New York State Museum. Bulletin 281, S. 3-19.

Smalley, Ian, J. und Unwin, David J. (1968) The formation and shape of drumlins and their distribution and orientation in drumlin fields. Journal of Glaciology, Vol. 7, 51, S. 377-390.

SuomenmaA VI (1924). Mikkelin lääni. Helsinki 1924, S. $1-247$.

Svensson, Harald und Frisèn, Rune (1964) Hällmorfologi och isrörelser inom ett alvarområde vid Degerhamn. Summary: Rock Morphology and Glacier Movement in a Limestone Area on the Island of Öland. Sveriges Geografiska Årsbok 1964. Årg. 40. Lund. The Swedish Geographical Yearbook 1964 (40), S. $19-30$.

TANnER, V. (1915) Studier öfver kvartärsystemet i Fennoskandias nordliga delar. III. (Résumé). Bulletin de la Commission géologique de Finlande 38, S. $1-815$.

- (1938) Die Oberflächengestaltung Finnlands. Bidrag till kännedom af Finlands natur och folk 86, S. 1-762.

TAylor, Frank Bursley (1906) Distribution of drumlins and its bearing on their origin. Bulletin of the Geological Society of America, Vol. 17, S. 726.

- (1931) Distribution of drumlins and its bearing on their origin. Bulletin of the Geological Society of America, Vol. 42, 1, S. 201.

Thornbury, William, D. (1969) Principles of Geomorphology. Second edition, S. 1-594.

Tolvanen, Viljo (1917) Eteläpohjanmaan ja Satakunnan välisen vedenjakajaseudun geomerfologiasta, S. 1177. Referat: Über die Geomorphologie des Wasserscheidengebiets zwischen Südostbottnien und Satakunta, S. 1-8. Meddelanden av Geografiska Före- ningen i Finland. Suomen Maantieteellisen yhdistyksen Julkaisuja XI, 1915-1920.

Vernon, Peter (1966) Drumlins and Pleistocene ice flow over the Ards Peninsula/Strangford Lough area, County Down, Ireland. Résumé und Zusammenfassung. Journal of Glaciology, Vol. 6, 45, S. 401-409.

VirkKala, K. (1948) Suomen geologinen yleiskartta. Lehti D4, Nurmes. Maalajikartan selitys. Suomen geologinen tutkimuslaitos, S. $1-101$.

- (1951) Glacial geology of the Suomussalmi area, East Finland. Bulletin de la Commission géologique de Finlande 155, S. 1-66.

- (1960) Geological map of Finland, 1:100 000. Sheets4421, 4422, 4423 -4441, 4424, Hyrynsalmi, Suomussalmi, Vuokkijärvi, Raate. Explanation to the map of superficial deposits. Geologinen tutkimuslaitos. The Geological Survey of Finland, S. 1-62.

- (1961) On the glacial geology of the Hämeenlinna region, southern Finland. Bulletin de la Commission géologique de Finland 196, S. 215-242.

- (1969) Suomen geologinen kartta. Geological map of Finland. Lehti - Sheet 2131, Hämeenlinna. Maaperäkartan selitys. Explanation to the map of Quaternary deposits. Summary: Explanatory text to the map of Quaternary deposits, Geologinen tutkimuslaitos. The Geological Survey of Finland. S. 1-69.

Wilkman, W. W. (1938) Suomen geologinen yleiskartta, Lehti C3, Kuopio. Kivilajikartan selitys. English summary. Suomen geologinen toimikunta, S. 1-171.

WiSNIEWSKr, Edward (1965) Formy drumlinowe okolic Gniewu. Drumlin forms in the Gniew Region. Przeglad Geograficzny t. XXXVII, 2. 1, 1965, S. 171-182.

Woldstedt, Paul (1961) Das Eiszeitalter. Grundlinien einer Geologie des Quartärs. I. Stuttgart, S. 1-374.

Wright, H. E., JR. (1957) Stone orientation in Wadena Drumlin field, Minnesota. Geografiska Annaler 39, 1, S. $19-31$.

Wright, W. B. (1912) The Drumlin Topography of South Donegal. The Geological Magazine. New Series, Vol. 9, III, S. 153-159.

Upham, WARREN (1889) The Structure of drumlins. Proceedings of the Boston Society of Natural History. Vol. XXIV, Part II. Dec 1888-May 1889, Boston 1889, S. $228-242$.

Manuskript eingegangen am 30. November 1970. 